\title{
A statistical analysis of the magnetic structure of CP stars
}

\author{
S. Bagnulo ${ }^{1}$, M. Landi Degl’Innocenti ${ }^{2}$, M. Landolfi ${ }^{3}$, and G. Mathys ${ }^{1}$ \\ 1 European Southern Observatory, Alonso de Cordova 3107, Vitacura, Santiago, Chile \\ e-mail: sbagnulo@eso.org, gmathys@eso.org \\ 2 C.N.R., Istituto di Radioastronomia, Sezione di Firenze, Largo E. Fermi 5, 50125 Firenze, Italy \\ e-mail: mlandi@arcetri.astro.it \\ 3 Istituto Nazionale di Astrofisica, Osservatorio Astrofisico di Arcetri, Largo E. Fermi 5, 50125 Firenze, Italy \\ e-mail: landolfi@arcetri.astro.it
}

Received 16 May 2002 / Accepted 6 August 2002

\begin{abstract}
We present the results of a statistical study of the magnetic structure of upper main sequence chemically peculiar stars. We have modelled a sample of 34 stars, assuming that the magnetic morphology is described by the superposition of a dipole and a quadrupole field, arbitrarily oriented. In order to interpret the modelling results, we have introduced a novel set of angles that provides one with a convenient way to represent the mutual orientation of the quadrupolar component, the dipolar component, and the rotation axis. Some of our results are similar to what has already been found in previous studies, e.g., that the inclination of the dipole axis to the rotation axis is usually large for short-period stars and small for long-period ones - see Landstreet \& Mathys (2000). We also found that for short-period stars (approximately $P<10$ days) the plane containing the two unit vectors that characterise the quadrupole is almost coincident with the plane containing the stellar rotation axis and the dipole axis. Long-period stars seem to be preferentially characterised by a quadrupole orientation such that the planes just mentioned are perpendicular. There is also some loose indication of a continuous transition between the two classes of stars with increasing rotational period.
\end{abstract}

Key words. stars: magnetic fields - polarization - stars: chemically peculiar

\section{Introduction}

Magnetic chemically peculiar $(\mathrm{CP})$ stars of the upper main sequence represent an astrophysical laboratory for the study of the interplay between magnetic field and the stellar photospheric plasma.

The magnetic field of $\mathrm{CP}$ stars is relatively strong (up to a few tens of $\mathrm{kG}$ ) and is characterised by a smooth geometry, thus it can be easily detected via spectropolarimetric techniques. At the same time, magnetic $\mathrm{CP}$ stars are not rare objects (they represent about $10 \%$ of all A and B stars), which makes it possible to observe how magnetic fields affect the stellar photospheres in many different situations (i.e., in stars of different age, mass, temperature, etc.). It appears for instance that magnetic fields tend to be stronger in hotter than in cooler stars (Landstreet 1992; Mathys et al. 1997; Hubrig et al. 2000). The presence of a magnetic field is generally associated with a non-homogeneous distribution of the chemical elements (both horizontally and vertically), and long rotational periods (up to several decades). Studying these phenomena, in particular the way various stellar features correlate with the magnetic field, may permit us to improve our knowledge of stellar astrophysics in general, as it is reasonable to hypothesise that those phenomena that are so

Send offprint requests to: S. Bagnulo, e-mail: sbagnulo@eso.org pronounced (thus so well observed) in magnetic CP stars play also some role in "normal" upper main sequence stars.

As a matter of fact, most of the physics that we could learn from the study of CP stars is not yet understood. One of the fundamental issues that still needs to be clarified is the origin of the magnetic field. Hints to the solution of this problem could come from more statistical information on the magnetic field geometries. In particular it would be important to know if there exist "typical" magnetic structures, and if there are relationships between magnetic fields and rotational velocity. There is an obvious need for constraints from observations and constraints obtained from secure modelling results.

Some important steps have been accomplished in the last few years, and information about the magnetic strength of CP stars has been derived from simple attempts to correlate magnetic field measurements with various stellar features. For sharp-lined stars, Mathys et al. (1997) found that the mean field modulus, averaged through the rotational cycle, is typically in the interval between 3 and $9 \mathrm{kG}$, and that the $3 \mathrm{kG}$ lower end represents in fact a sharp cutoff of the distribution. (However, it should be noted that, for mean field modulus values less than $1.5-2 \mathrm{kG}$, Zeeman splitting is typically smaller than the intrinsic line width, so that the mean field modulus cannot be measured. Therefore, it is more appropriate to say that the evidence found by Mathys et al. 1997 is for a shortage of sharp-lined 
stars with mean field modulus between about 1.5 and $3 \mathrm{kG}$.) Mathys et al. (1997) also discovered that the mean field modulus of stars with rotation period longer than $150 \mathrm{~d}$ is never stronger than $7.5 \mathrm{kG}$, which suggests a sort of anti-correlation between star's rotation period and mean field modulus. Finally, one of the most important findings about the magnetic structure of chemically peculiar stars was recently obtained from a simple modelling of the mean longitudinal field and the mean field modulus. Assuming an axisymmetric model described by the superposition of a dipole plus colinear quadrupole and octupole, Landstreet \& Mathys (2000) found that slow rotating CP stars (i.e., stars with rotational period longer than one month) have a magnetic axis tilted at a small angle $\left(\lesssim 30^{\circ}\right)$ with respect to the rotation axis. It is clear that these kinds of information are extremely valuable in order to explain the formation of the magnetic field (see Moss 2001), and also to understand all those phenomena that are most likely connected to the presence of a magnetic field, such as, e.g., the loss of angular momentum (Stępień \& Landstreet 2002).

In the past, it has been commonly assumed that magnetic fields of CP stars have a quasi-dipolar morphology that can be described in terms of a low-order and axisymmetric multipolar expansion. This assumption was based on the fact that in several cases an axisymmetric morphology was indeed found sufficient to interpret the available observations - mainly represented by determinations of mean longitudinal field. Recent developments of the observing techniques (including spectropolarimetry of all Stokes profiles, see Wade et al. 2000a), followed by attempts to interpret the newly obtained data, point to evidence for ubiquitous departures of the magnetic structures from axisymmetric geometries (e.g., Mathys et al. 1997; Bagnulo et al. 1999, hereafter Paper II; Bagnulo et al. 2001). From the theoretical point of view we note that - should the field be either a fossil relic or dynamo generated - there is no reason why stellar magnetic fields should have cylindrical symmetries. In fact, there is no reason why a fossil field should have an axisymmetric geometry, and it is theoretically predicted that dynamo generated fields do develop a non-axisymmetric geometry (Moss 2001). These considerations prompt for further searches for statistical properties of the magnetic structures of CP stars by making use of a modelling technique not limited by simplifying approximations on the magnetic geometry. A fully adequate modelling technique based on the inversion of Stokes profiles may exist: the Zeeman Doppler Imaging (ZDI; see Piskunov \& Kochukhov 2002). However, only few stars have been modelled in detail through the inversion of Stokes profiles (e.g., $\alpha^{2}$ CVn, see Kochukhov et al. 2002). We are lacking sufficient high quality data (in terms of spectral and phase coverage, spectral resolution and signal to noise ratio) to be able to perform a statistically meaningful analysis based on ZDI. On the other hand, we have at our disposal a large number of magnetic field observations obtained through measurements of Zeeman splitting and of the low-order moments of Stokes profiles $I$ and $V$, such as the aforementioned mean field modulus and longitudinal field, but also the crossover and the mean quadratic field. These kinds of data (referred to as "magnetic observables" - see, e.g., Bagnulo et al. 2000, hereafter Paper III) can be obtained from comparatively low-resolution, low signal-to-noise ratio spectra, even, in certain cases, from spectra recorded with photographic plates, making it possible to extract information on the stellar magnetic geometry from observational data that are not suitable for application of straight inversion techniques.

In this paper we present a statistical investigation of the magnetic structure of CP stars based on the interpretation of the magnetic observables, under the assumption that the stellar magnetic field is described by the superposition of a dipole and a quadrupole field, arbitrarily oriented. Although it seems unlikely that such a low-order multipolar expansion can accurately describe the real magnetic configurations of CP stars (see, e.g., Bagnulo et al. 2001), it is reasonable to hope that the analysis of a large sample of stars can still provide us with some insight into the problem of whether there exist statistical properties of the magnetic structures of CP stars other than those that have been already found.

A formalism for the description of the magnetic field in terms of dipole plus quadrupole (arbitrarily oriented) was presented by Bagnulo et al. (1996) and Landolfi et al. (1998) - hereafter Paper I - and modelling results for individual stars were presented, e.g., in Paper II and Bagnulo \& Landolfi (1999). Preliminary statistical results were presented by Bagnulo (2001), who found for instance that in faster rotating stars, one of the unit vectors that define the quadrupolar component is generally aligned to the rotation axis. Then it was realised that a set of new angles could be adopted in order to obtain a clearer description of the statistical results, in particular by explicitly referring to the orientation of the plane defined by the quadrupolar component with respect to the dipole axis and the rotation axis. Such angles are introduced in Sect. 2, which also presents the guidelines for the statistical analysis performed in Sects. 3 and 4. In Sect. 5 we summarise and briefly discuss our results.

\section{Analysis of observations: Generalities}

We tried to interpret the observations of mean longitudinal field, crossover, mean quadratic field - as defined by Mathys (1995a, 1995b) - and mean field modulus for a sample of 34 magnetic CP stars in terms of the oblique rotator model with a dipole plus (non linear) quadrupole field. For such model, the magnetic field vector at the point $\boldsymbol{r}$ of the stellar surface is given by (see Paper I)

$\mathcal{B}(\boldsymbol{r})=\mathcal{B}_{\mathrm{d}}(\boldsymbol{r})+\mathcal{B}_{\mathrm{q}}(\boldsymbol{r})$

where

$$
\begin{aligned}
\mathcal{B}_{\mathrm{d}}(\boldsymbol{r})= & -\frac{B_{\mathrm{d}}}{2}\left[\boldsymbol{u}-3 \frac{\boldsymbol{u} \cdot \boldsymbol{r}}{R_{*}^{2}} \boldsymbol{r}\right] \\
\mathcal{B}_{\mathrm{q}}(\boldsymbol{r})= & -\frac{B_{\mathrm{q}}}{2}\left[\frac{\boldsymbol{u}_{\mathbf{2}} \cdot \boldsymbol{r}}{R_{*}} \boldsymbol{u}_{\mathbf{1}}+\frac{\boldsymbol{u}_{\mathbf{1}} \cdot \boldsymbol{r}}{R_{*}} \boldsymbol{u}_{\mathbf{2}}\right. \\
& \left.+\left(\frac{\boldsymbol{u}_{\mathbf{1}} \cdot \boldsymbol{u}_{\mathbf{2}}}{R_{*}}-5 \frac{\left(\boldsymbol{u}_{\mathbf{1}} \cdot \boldsymbol{r}\right)\left(\boldsymbol{u}_{\mathbf{2}} \cdot \boldsymbol{r}\right)}{R_{*}^{3}}\right) \boldsymbol{r}\right] .
\end{aligned}
$$

Here $R_{*}$ is the star's radius; $B_{\mathrm{d}}$ and $B_{\mathrm{q}}$ are the dipole and quadrupole amplitude, respectively; the unit vector $\boldsymbol{u}$ specifies 
the direction of the dipole axis; the unit vectors $\boldsymbol{u}_{1}, \boldsymbol{u}_{2}$ specify the orientation of the quadrupole.

The four magnetic observables are denoted in this paper by the notations

$$
\begin{array}{ll}
\left\langle\mathcal{B}_{z}\right\rangle & \rightarrow \text { mean longitudinal field } \\
v_{\mathrm{e}} \sin i\left\langle d \mathcal{B}_{z}\right\rangle & \rightarrow \text { crossover } \\
\left\langle\mathcal{B}^{2}+\mathcal{B}_{z}^{2}\right\rangle^{1 / 2} & \rightarrow \text { mean quadratic field } \\
\langle\mathcal{B}\rangle & \rightarrow \text { mean field modulus. }
\end{array}
$$

The expressions relating these quantities to the model parameters were derived, under certain assumptions on the stellar atmosphere, by Bagnulo et al. (1996), and rewritten in a more compact form in Papers I and III, respectively. An inversion procedure for recovering the model parameters from observations of $\left\langle\mathcal{B}_{z}\right\rangle, v_{\mathrm{e}} \sin i\left\langle d \mathcal{B}_{z}\right\rangle$, and $\left\langle\mathcal{B}^{2}+\mathcal{B}_{z}^{2}\right\rangle^{1 / 2}$ was described extensively in Papers I and II, and subsequently generalized in Paper III to include observations of $\langle\mathcal{B}\rangle$, measurements of projected equatorial velocity, and estimates of the stellar radius. For this work we used the numerical code described in Paper III, with the only difference that linear polarization observations, not available for most of the stars in our sample, were disregarded.

The magnetic geometry of the dipole plus quadrupole model is illustrated in detail in Paper I. We simply recall that such model depends on the following 10 parameters:

- $B_{\mathrm{d}}$ and $B_{\mathrm{q}}$;

- $v_{\mathrm{e}}$, the stellar equatorial velocity;

$-i$, the inclination of the stellar rotation axis to the line of sight;

$-\beta$, the angle between the direction $\boldsymbol{u}$ and the rotation axis;

$-\beta_{1}$ and $\beta_{2}$, the analogues of $\beta$ for the directions $\boldsymbol{u}_{1}, \boldsymbol{u}_{2}$;

- $\gamma_{1}$ and $\gamma_{2}$, the azimuthal angles of $\boldsymbol{u}_{1}, \boldsymbol{u}_{2}$ about the rotation axis;

$-f_{0}$, the "reference" rotational phase of the model (see Fig. 1 of Paper I).

The angles $i, \beta, \beta_{1}, \beta_{2}$ range from $0^{\circ}$ to $180^{\circ}$, while $\gamma_{1}, \gamma_{2}, f_{0}$ range from $0^{\circ}$ to $360^{\circ}$. The rotational period $P$ and the limbdarkening constant $u$, which also affect the expressions of the magnetic quantities, are taken as fixed. In this work we adopted $u=0.5$ for all stars, but the results of the inversion procedure are only slightly influenced by the $u$ value, as discussed in Papers I-III.

As explained in Paper III, observations of $\left\langle\mathcal{B}_{z}\right\rangle$, $v_{\mathrm{e}} \sin i\left\langle d \mathcal{B}_{z}\right\rangle,\left\langle\mathcal{B}^{2}+\mathcal{B}_{z}^{2}\right\rangle^{1 / 2}$, and $\langle\mathcal{B}\rangle$, do not allow one to distinguish between two magnetic configurations symmetrical about the plane containing the rotation axis and the dipole axis. Such configurations are characterised by the same values of $B_{\mathrm{d}}, B_{\mathrm{q}}, v_{\mathrm{e}}, \gamma_{1}, \gamma_{2}, f_{0}$, while the remaining angles are related by

$$
\begin{array}{cccc}
(i, & \beta, & \beta_{1}, & \left.\beta_{2}\right) \\
\left(180^{\circ}-i,\right. & 180^{\circ}-\beta, & 180^{\circ}-\beta_{1}, & \left.180^{\circ}-\beta_{2}\right) .
\end{array}
$$

On the other hand, as shown in Paper I, exchange of directions $\boldsymbol{u}_{1}$ and $\boldsymbol{u}_{2}$, as well as inversion of both $\left(\boldsymbol{u}_{1} \rightarrow-\boldsymbol{u}_{1}, \boldsymbol{u}_{2} \rightarrow-\boldsymbol{u}_{2}\right)$, leaves the magnetic configuration unchanged. This means that the sets

$$
\begin{aligned}
& \left(\beta_{1}, \beta_{2}, \gamma_{1}, \gamma_{2}\right) \\
& \left(\beta_{2}, \beta_{1}, \gamma_{2}, \gamma_{1}\right)
\end{aligned}
$$

and

$$
\begin{array}{cccr}
\left(\beta_{1},\right. & \beta_{2}, & \gamma_{1}, & \left.\gamma_{2}\right) \\
\left(180^{\circ}-\beta_{1},\right. & 180^{\circ}-\beta_{2}, & 180^{\circ}+\gamma_{1}, & \left.180^{\circ}+\gamma_{2}\right)
\end{array}
$$

describe the same magnetic configuration.

A convenient way to visualise the quadrupole orientation is to introduce the plane containing the unit vectors $\boldsymbol{u}_{1}, \boldsymbol{u}_{2}$ and its pole $\mathrm{Q}$, defined by

$\boldsymbol{u}_{\mathrm{Q}}=\frac{\boldsymbol{u}_{1} \times \boldsymbol{u}_{2}}{\left|\boldsymbol{u}_{1} \times \boldsymbol{u}_{2}\right|}$

if $\boldsymbol{u}_{1} \times \boldsymbol{u}_{2} \neq 0$, undefined otherwise (see Fig. 1). The point Q is characterised by the angles $\beta_{\mathrm{Q}}$ and $\gamma_{\mathrm{Q}}$, while the orientation of $\boldsymbol{u}_{1}, \boldsymbol{u}_{2}$ in their plane is characterised by the angles $\chi_{1}$ and $\chi_{2}$ reckoned from the "reference" semi-meridian (through $\mathrm{R}$ and $\mathrm{D}$ ), or, equivalently, by their linear combinations $\chi_{\text {- }}$ and $\chi_{+}$defined by

$$
\begin{aligned}
& \chi_{-}= \begin{cases}\left|\chi_{1}-\chi_{2}\right| & \text { if }\left|\chi_{1}-\chi_{2}\right| \leq 180^{\circ} \\
360^{\circ}-\left|\chi_{1}-\chi_{2}\right| & \text { if }\left|\chi_{1}-\chi_{2}\right|>180^{\circ}\end{cases} \\
& \chi_{+}= \begin{cases}\left(\chi_{1}+\chi_{2}\right) / 2 & \text { if }\left|\chi_{1}-\chi_{2}\right| \leq 180^{\circ} \\
\left(\chi_{1}+\chi_{2}\right) / 2+180^{\circ} & \text { if }\left|\chi_{1}-\chi_{2}\right|>180^{\circ} \\
& \text { and }\left(\chi_{1}+\chi_{2}\right)<360^{\circ} \\
\left(\chi_{1}+\chi_{2}\right) / 2-180^{\circ} & \text { if }\left|\chi_{1}-\chi_{2}\right|>180^{\circ} \\
& \text { and }\left(\chi_{1}+\chi_{2}\right) \geq 360^{\circ},\end{cases}
\end{aligned}
$$

which represent the "width" and "barycentre" of the unit vectors $\boldsymbol{u}_{1}, \boldsymbol{u}_{2}$, respectively. In the following we will refer to the angles $\beta_{\mathrm{Q}}, \gamma_{\mathrm{Q}}, \chi_{-}, \chi_{+}$rather than to $\beta_{1}, \beta_{2}, \gamma_{1}, \gamma_{2}$.

The stars of our sample are listed in Table 1 . The columns contain the following data (from left to right): a reference number used in this paper; the HD number of the object; a second identifier for the star; the stellar rotational period in days; the number of observations used in this work for each of the four magnetic observables; the observational value of the projected equatorial velocity; the estimated value of the stellar radius. The meaning of the last three columns (labelled with $g, p 1$, and $p 2$ ) will be explained later.

Most magnetic field measurements were taken from Mathys (1994) (mean longitudinal field), Mathys (1995a) (crossover), Mathys (1995b) (mean quadratic field, but the determinations were revised as explained in Mathys et al., in preparation), Mathys \& Hubrig (1997) (longitudinal field, crossover, quadratic field), Mathys et al. (1997) (mean field modulus). We also considered additional measurements, to be published by Mathys et al. (in preparation), most of which were already used by Landstreet \& Mathys (2000). Additional mean longitudinal field data were taken from Bagnulo et al. (2001), Borra \& Landstreet (1977, 1978), Hildebrandt et al. (1997), Hill et al. (1998), Leone et al. (2000), Leone \& Catanzaro (2001), Preston \& Stępień (1968), Wade et al. (1996, 1997, 2000a, 2000b, 2000c). Further mean field modulus data were taken from Huchra (1972), and Preston (1969).

The adopted value of the rotational period and estimates of the projected equatorial velocity come mainly from the same references as for the magnetic data. However, in many 
Table 1. The star' sample analysed in this work. A dash in columns $\left\langle d B_{z}\right\rangle$ (an abbreviation for $v_{\mathrm{e}} \sin i\left\langle d \mathcal{B}_{z}\right\rangle$ ), $\left\langle B B_{z}\right\rangle$ (an abbreviation for $\left.\left\langle\mathcal{B}^{2}+\mathcal{B}_{z}^{2}\right\rangle^{1 / 2}\right),\langle\mathcal{B}\rangle$, and $v_{\mathrm{e}} \sin i$, means that no observation was available. The last three columns show the value of the reduced $\chi^{2}$ corresponding to the best-fit model, as derived from 3 different types of data analysis, i.e., considering all observed quantities (Col. $g$ ), considering all observed quantities but $\left\langle\mathcal{B}^{2}+\mathcal{B}_{z}^{2}\right\rangle^{1 / 2}$ (Col. $p 1$ ), and considering all observed quantities but $\langle\mathcal{B}\rangle$ (Col. $p 2$ ). A blank in columns $p 1$ and $p 2$ means that the star does not pertain to the sub-sample (see Sect. 4).

\begin{tabular}{|c|c|c|c|c|c|c|c|c|c|c|c|c|}
\hline No. & HD & Other Identifier & $P$ & $\left\langle\mathcal{B}_{z}\right\rangle$ & $\left\langle d B_{z}\right\rangle$ & $\left\langle B B_{z}\right\rangle$ & $\langle\mathcal{B}\rangle$ & $v_{\mathrm{e}} \sin i$ & $R_{*}$ & $g$ & $p 1$ & $p 2$ \\
\hline 1 & 2453 & GR And & 519.5 & 33 & 6 & 6 & 14 & - & 2.97 & 2.08 & 2.25 & 1.57 \\
\hline 2 & 12288 & V540 Cas & 34.91 & 20 & - & - & 28 & - & 3.28 & 1.75 & 1.75 & \\
\hline 3 & 14437 & $\mathrm{AG}+42247$ & 26.87 & 36 & - & - & 31 & - & 3.08 & 1.96 & 1.96 & \\
\hline 4 & 24712 & DO Eri & 12.4592 & 82 & 7 & 5 & - & 5.6 & 1.83 & 3.56 & & 3.56 \\
\hline 5 & 51684 & CD-40 2796 & 371.0 & 7 & 4 & 7 & 10 & - & 3.58 & 0.93 & 0.59 & 0.68 \\
\hline 6 & 61468 & CD-27 4341 & 322.0 & 5 & 5 & 5 & 15 & - & 4.16 & $>8$ & 6.29 & 1.57 \\
\hline 7 & 65339 & 53 Cam & 8.02681 & 66 & 6 & - & 36 & 13.0 & 2.56 & 4.21 & 4.21 & \\
\hline 8 & 70331 & CD-47 3803 & 1.99812 & 16 & 16 & 16 & 38 & - & - & 1.46 & 1.58 & 1.34 \\
\hline 9 & 75445 & CD-38 4907 & 6.29100 & 3 & 3 & 3 & 11 & - & 2.34 & 1.54 & 0.87 & \\
\hline 10 & 81009 & KU Hya & 33.9840 & 13 & 13 & 13 & 42 & - & 2.61 & 1.95 & 1.32 & 1.38 \\
\hline 11 & 83368 & HR 3831 & 2.851982 & 12 & 12 & 11 & - & 32.6 & 2.23 & 2.08 & & 2.08 \\
\hline 12 & 93507 & CD-67 955 & 568.6 & 12 & 12 & 12 & 30 & - & 3.73 & 3.70 & 1.59 & 2.03 \\
\hline 13 & 94660 & KQ Vel & 2764.0 & 12 & 12 & 12 & 25 & - & 3.15 & $>8$ & 1.60 & 1.39 \\
\hline 14 & 96446 & CD-59 3544 & 0.85137 & 9 & 9 & 9 & - & 10.0 & 6.43 & 2.98 & & 2.98 \\
\hline 15 & 116114 & BD-17 3829 & 26.71 & 7 & 7 & 7 & 24 & - & 3.06 & 1.72 & 1.46 & 2.24 \\
\hline 16 & 116458 & HR 5049 & 148.39 & 20 & 20 & 20 & 21 & - & 3.54 & 2.42 & 1.89 & 2.01 \\
\hline 17 & 119419 & HR 5158 & 2.60090 & 22 & 22 & 22 & - & 34.8 & 2.23 & 1.71 & & 1.71 \\
\hline 18 & 125248 & CS Vir & 9.2954 & 31 & 21 & 21 & - & 8.0 & 1.97 & 1.85 & & 1.85 \\
\hline 19 & 126515 & Preston's star & 129.95 & 25 & 19 & 19 & 22 & - & 2.35 & 4.56 & 2.99 & 4.35 \\
\hline 20 & 137509 & NN Aps & 4.4916 & 14 & 14 & 14 & - & 28.1 & 3.59 & 1.20 & & 1.20 \\
\hline 21 & 137909 & $\beta \mathrm{CrB}$ & 18.4868 & 51 & 21 & 21 & 46 & 3.5 & 3.25 & 2.09 & 2.15 & 2.45 \\
\hline 22 & 142070 & AG-00 2049 & 3.37180 & 13 & 13 & 13 & 24 & 13.5 & 5.42 & $>8$ & 3.19 & 2.72 \\
\hline 23 & 144897 & CD-40 10236 & 48.6200 & 13 & 13 & 13 & 28 & - & 3.97 & 2.53 & 1.70 & 1.93 \\
\hline 24 & 147010 & V933 Sco & 3.920676 & 19 & 19 & 19 & - & 22.1 & 2.85 & 1.68 & & 1.68 \\
\hline 25 & 153882 & HR 6326 & 6.00890 & 17 & 17 & 17 & - & 21.0 & 3.81 & 2.17 & & 2.17 \\
\hline 26 & 175362 & V686 CrA & 3.67375 & 30 & 30 & 29 & - & 15.0 & 2.68 & 3.97 & & 3.97 \\
\hline 27 & 187474 & V3961 Sgr & 2364.0 & 20 & 20 & 20 & 40 & - & 2.93 & 3.82 & 3.59 & 3.36 \\
\hline 28 & 188041 & V1291 Aql & 223.95 & 77 & 8 & - & 18 & - & 3.14 & 1.41 & 1.41 & \\
\hline 29 & 192678 & V1372 Cyg & 6.4186 & 14 & - & - & 34 & - & 3.05 & 2.17 & 2.17 & \\
\hline 30 & 200311 & V2200 Cyg & 52.0703 & 24 & - & - & 35 & 9.0 & 2.18 & 2.37 & 2.37 & \\
\hline 31 & 208217 & BD Ind & 8.44473 & 8 & 8 & 8 & 38 & 15.3 & 2.60 & 1.58 & 1.73 & 1.64 \\
\hline 32 & 215441 & Babcock's star & 9.4871 & 14 & - & - & 11 & 5.0 & 2.45 & 2.51 & 2.51 & \\
\hline 33 & 318107 & V970 Sco & 9.70870 & 6 & 6 & 6 & 36 & - & 2.46 & 3.37 & 2.15 & 3.55 \\
\hline 34 & 335238 & $\mathrm{AG}+292421$ & 48.7 & 3 & 3 & 3 & 18 & - & 3.69 & 1.52 & 1.15 & \\
\hline
\end{tabular}

cases, the rotational period was re-computed through a leastsquare technique based on a second-order Fourier expansion to the longitudinal field and mean field modulus measurements. Estimates for the stellar radius $R_{*}$ were taken from North (1998) and Hubrig et al. (2000). Additional $R_{*}$ estimates were kindly provided to us by North (2001, private communication).

The aim of this study is not so much to find the "best" magnetic model for each individual star as to characterise the sample as a whole in order to see whether there are any "preferred" magnetic geometries (in the framework of the assumed dipole plus quadrupole model) and/or correlations between magnetic model and the rotational period.
The main difficulty underlying an analysis of this kind is due to the fact that the inversion algorithm usually produces, for a given star, several different models, i.e., several sets of parameters, corresponding to local minima of the $\chi^{2}$ hypersurface. As discussed in Papers I, II, and III, this is primarily due to the relatively large observational errors. For some stars the $\chi^{2}$ values at the various minima are very different from each other, but in other cases these values are not so different. Therefore, it looks rather unsafe to restrict the statistical analysis to best-fit models (i.e., those corresponding to the absolute $\chi^{2}$ minimum). Furthermore, even if this restriction were applied, the magnetic 


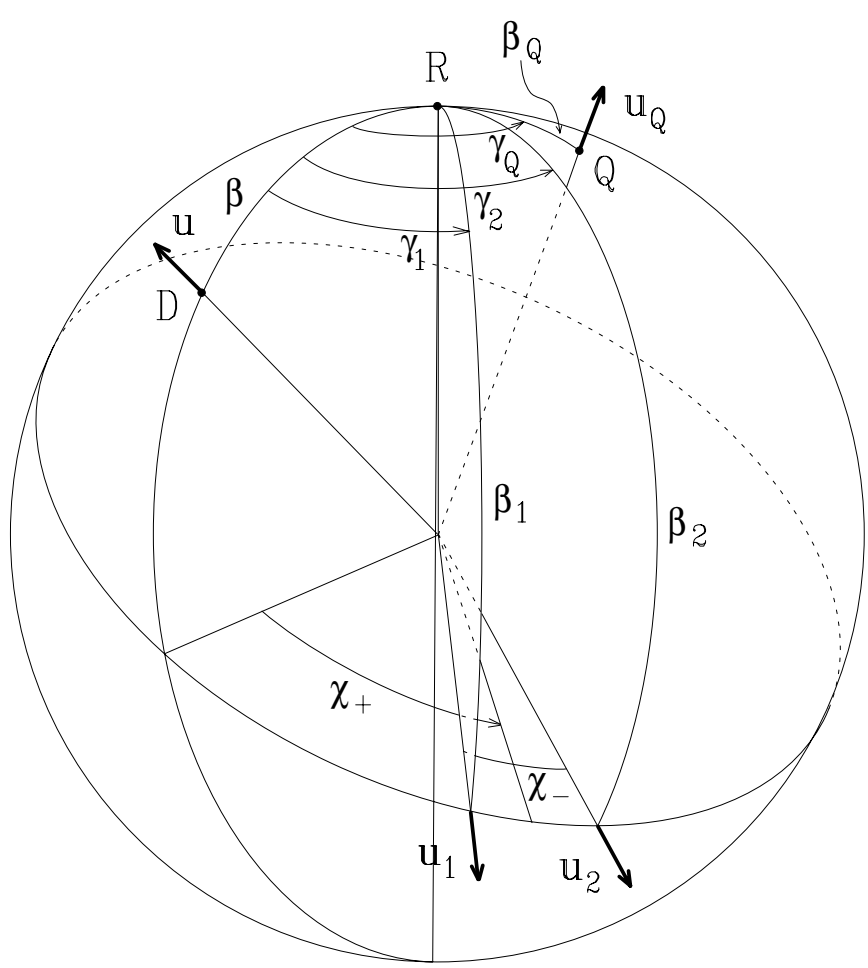

Fig. 1. Geometry of the dipole plus quadrupole model. The rotation axis intersects the stellar surface at $\mathrm{R}$, and makes an angle $i$ (not shown in the figure) with the line of sight. $\mathrm{D}$ is the positive pole of the dipole. $\mathrm{Q}$ is the pole of the quadrupole plane defined by the unit vectors $\boldsymbol{u}_{1}$ and $\boldsymbol{u}_{2}$.

models of the individual stars could not be considered equally reliable, being characterised by different $\chi^{2}$ values.

The most natural way to deal with these problems is to "weight" the parameter sets associated with the $\chi^{2}$ minima. In this work we introduced a weight $\rho_{j}$ for each star, defined by

$\rho_{j}=\frac{1}{\left(\chi_{\min }^{2}\right)_{j}} \quad(j=1, \ldots, 34)$,

where $\chi_{\min }^{2}$ is the value of the reduced $\chi^{2}$ corresponding to the absolute minimum, and a weight $\rho_{j k}$ for the $k$ th parameter set, defined by

$\rho_{j k}=\frac{c_{j}}{\left[\left(\chi_{j k}^{2}\right)\right]^{p}} \quad(k=1, \ldots, m(j))$,

where $m(j)$ is the "multiplicity" of the $j$ th star, i.e., the number of minima in the $\chi^{2}$ hypersurface. The quantity $c_{j}$ is determined by requiring that

$\sum_{k=1}^{m(j)} \rho_{j k}=\rho_{j}$.

The figures presented in the following were obtained for $p=2$, but the value $p=1$ leads to quite similar results.

An additional difficulty concerns the type of observations to be used for the modelling. In particular, as noticed already in previous works (e.g., Paper III; Landstreet \& Mathys 2000), for many stars, $\left\langle\mathcal{B}^{2}+\mathcal{B}_{z}^{2}\right\rangle^{1 / 2}$ and $\langle\mathcal{B}\rangle$ measurements are not consistent among themselves. For this reason we performed separate investigations, by using all observations together (Sect. 3),
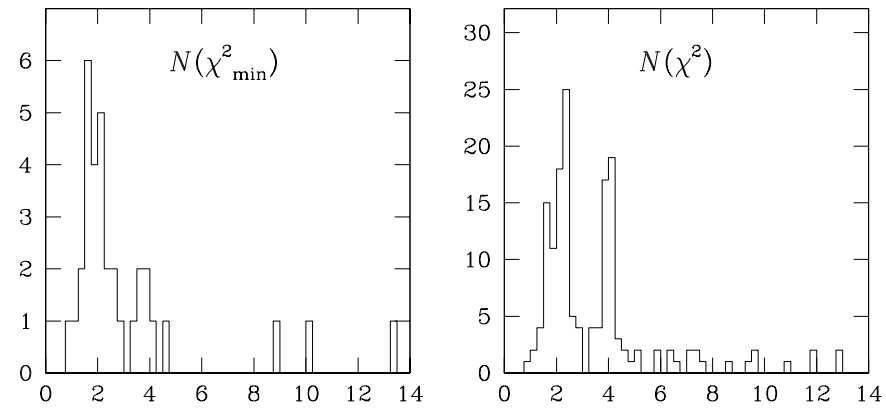

Fig. 2. Left: distribution of the minimum $\chi^{2}$ values for the magnetic models of the 34 stars of the sample (see Col. $g$ of Table 1). Right: same for the models corresponding to all (absolute and relative) $\chi^{2}$ minima. Further absolute and relative minima with larger $\chi^{2}$ values are not shown in the figure. See text for details.

and by disregarding either $\left\langle\mathcal{B}^{2}+\mathcal{B}_{z}^{2}\right\rangle^{1 / 2}$ or $\langle\mathcal{B}\rangle$ observations (Sect. 4). The former kind of analysis, i.e., based on all observations together, has the obvious advantage that all the information available for a given star is used in the modelling; moreover, all the 34 stars listed in Table 1 can be considered. The latter kind of analysis may help to reveal if some results are likely biased by systematic measurement errors.

\section{Analysis based on 4 magnetic observables}

In this section we present the results of the "global" analysis, i.e., based on all the observations ${ }^{1}$. Column $g$ of Table 1 shows the value of the reduced $\chi^{2}$ corresponding to the best fit - absolute minimum of the $\chi^{2}$ hypersurface. The same data is plotted, in the form of a histogram, in Fig. 2 (left). One can see that most of the best fits are characterised by $\chi_{\min }^{2} \simeq 2$, with a secondary group at $\chi_{\min }^{2} \lesssim 4$. However, for three stars - HD 61468 , HD 94660, and HD 142070 - no reasonable fit was found. This may be ascribed to inconsistencies between $\left\langle\mathcal{B}^{2}+\mathcal{B}_{z}^{2}\right\rangle^{1 / 2}$ and $\langle\mathcal{B}\rangle$ observations: in fact, if either of them is neglected, the $\chi^{2}$ value decreases considerably (see Cols. $p 1$ and $p 2$ of Table 1 ). However, we suspect that the magnetic morphology may be rather more complex than a dipole plus quadrupole field, especially in the case of HD 142070, which exhibits a strongly anharmonic mean field modulus curve. Those three stars were excluded from the analysis described in this section.

Figure 2 (right) shows the distribution of the reduced $\chi^{2}$ values for all the fits (corresponding either to the absolute minimum or to relative minima of the $\chi^{2}$ hypersurface). Altogether there are 168 minima, 12 of which with $\chi^{2}>14$. The relevant distribution is quite similar to that of the left panel, with a main group around $\chi^{2}=2$ and a secondary group at $\chi^{2} \simeq 4$; for larger values, the number of minima decreases rapidly. We decided to confine our study to models with $\chi^{2}<8$ : this leads to 147 models, associated with the 31 stars of the sample.

1 The magnetic observables considered in this section are in principle 4. However, since some stars lack observations of some kind (see Table 1), the modelling is actually based on 4,3 , or 2 observables, depending on the star. 

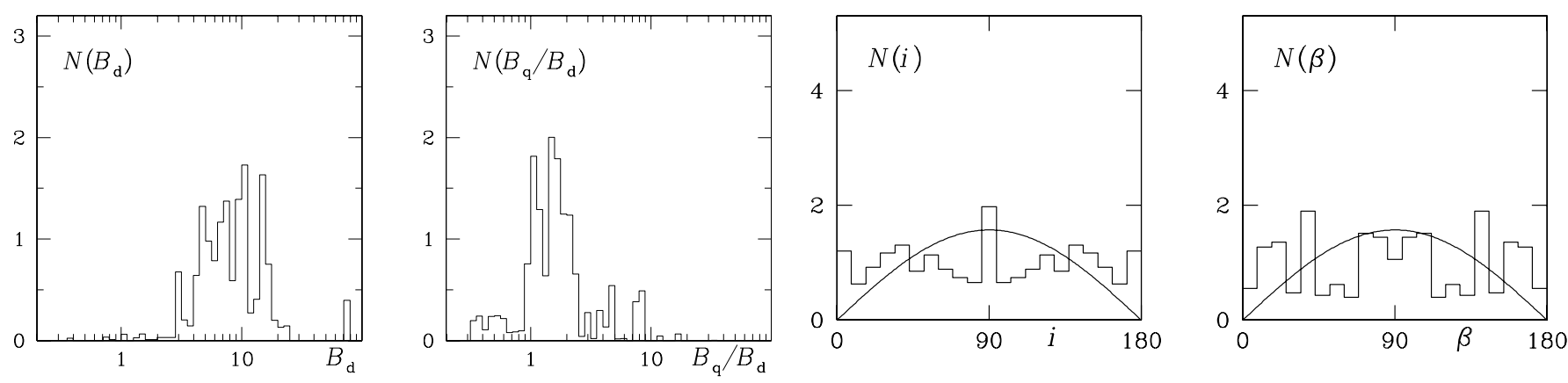

Fig. 3. Left: distribution of the dipole field amplitude. $B_{\mathrm{d}}$ is expressed in $\mathrm{kG}$. The isolated tooth on the far right is associated with Babcock's star (HD 215441, $B_{\mathrm{d}} \simeq 79 \mathrm{kG}$ ). Right: distribution of the ratio of quadrupole to dipole field amplitude.

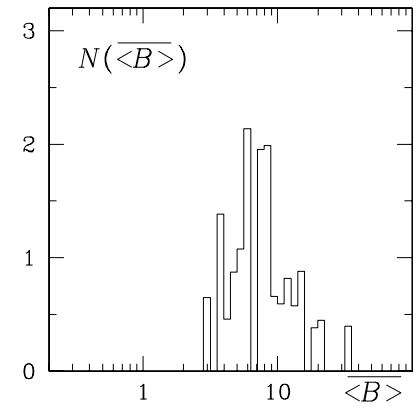

Fig. 4. Distribution of the phase-averaged mean field modulus $\overline{\langle\mathcal{B}\rangle}$ (expressed in $\mathrm{kG}$ ). The tooth on the right is Babcock's star.

Consider first the magnetic field strength. Figure 3 (left) shows the distribution of the dipole amplitude, $B_{\mathrm{d}}$. It is a rather regular, bell-shaped distribution peaking at something less than $10 \mathrm{kG}$; most models are characterised by $B_{\mathrm{d}}$ values ranging from $3 \mathrm{kG}$ to $20 \mathrm{kG}$. We recall that the histogram represents the $B_{\mathrm{d}}$ distribution weighted according to Eqs. (5)-(7): explicitly, the height of the histogram in the $i$ th interval is

$N_{i}=\sum_{j=1}^{31} \sum_{k=1}^{m(j)} \rho_{j k}^{(i)}$,

where $\rho_{j k}^{(i)}$ is given by Eq. (6) if the $B_{\mathrm{d}}$ value of the $k$ th model of the $j$ th star falls in the $i$ th interval, and is zero otherwise. The right panel of Fig. 3 shows the distribution of the ratio between quadrupole and dipole amplitude. It appears that the quadrupolar contribution is generally important, with $B_{\mathrm{q}}$ values varying approximately between $1 / 3$ and 10 times the $B_{\mathrm{d}}$ value. Finally, Fig. 4 shows the distribution of the phase-averaged mean field modulus,

$\overline{\langle\mathcal{B}\rangle}=\frac{1}{2 \pi} \int_{0}^{2 \pi}\langle B(f)\rangle \mathrm{d} f$

which quantifies the typical field strength for the stars in the sample. Contrary to $B_{\mathrm{d}}$ and $B_{\mathrm{q}}$, the value of $\overline{\langle\mathcal{B}\rangle}$ is - as expected - practically the same for all models of a given star: this explains why the histogram of Fig. 4 is less "scattered" than those of Fig. 3. Obviously, the quantity $\overline{\langle\mathcal{B}\rangle}$ is not (contrary to $B_{\mathrm{d}}$ and $B_{\mathrm{q}}$ ) intrinsic to the individual star, because it depends on the inclination of the rotation axis with respect to the line
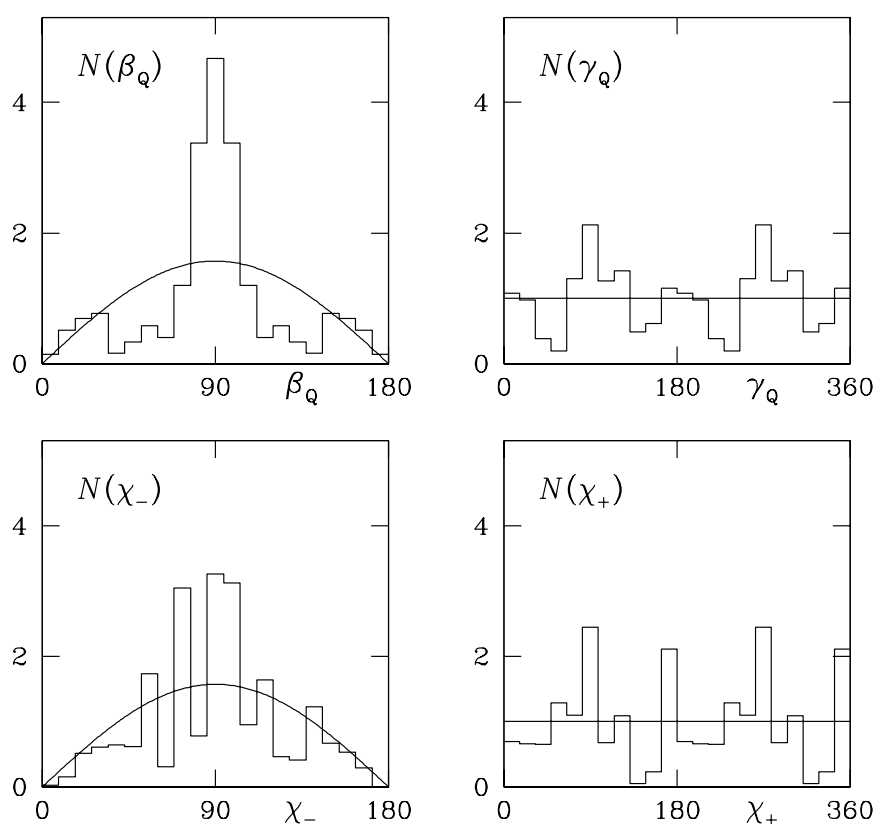

Fig. 5. Distributions of the angles specifying the magnetic geometry, compared with the relevant random distributions. Angles are expressed in degrees.

of sight (the angle $i$ ). We will comment further on this figure in Sect. 4.

Next we consider the distributions of the different angles. These are plotted in Fig. 5, together with the curves describing the relevant random distributions (sinusoidal variation for $i, \beta$, $\beta_{\mathrm{Q}}, \chi_{-}$; flat distribution for $\gamma_{\mathrm{Q}}$ and $\left.\chi_{+}\right)$. It should be noticed that the apparent symmetry characteristics of several histograms is just a consequence of the properties in Eqs. (2)-(4). The distributions of $i$ and $\beta$ are symmetrical about $90^{\circ}$ because of the degeneracy of Eq. (2), the distribution of $\beta_{\mathrm{Q}}$ because of Eq. (3); similarly, the distributions of $\gamma_{\mathrm{Q}}$ and $\chi_{+}$are invariant under a $180^{\circ}$ shift owing to Eqs. (2) and (4), respectively. By contrast, the distribution of $\chi_{-}$is unaffected by those properties (no symmetry characteristics).

It is obvious from Fig. 5 that most distributions, and especially that of $\beta_{\mathrm{Q}}$, are very different from the relevant random distributions. In order to quantify such difference, we decided to apply the Kolmogorov-Smirnov test (see, e.g., Press et al. 1986) to each distribution. As is well known, the test is based on the measurement of the maximum distance, $D$, between the cumulative distribution of the observational data and the corresponding theoretical distribution. Such distributions are shown in Fig. 6. 

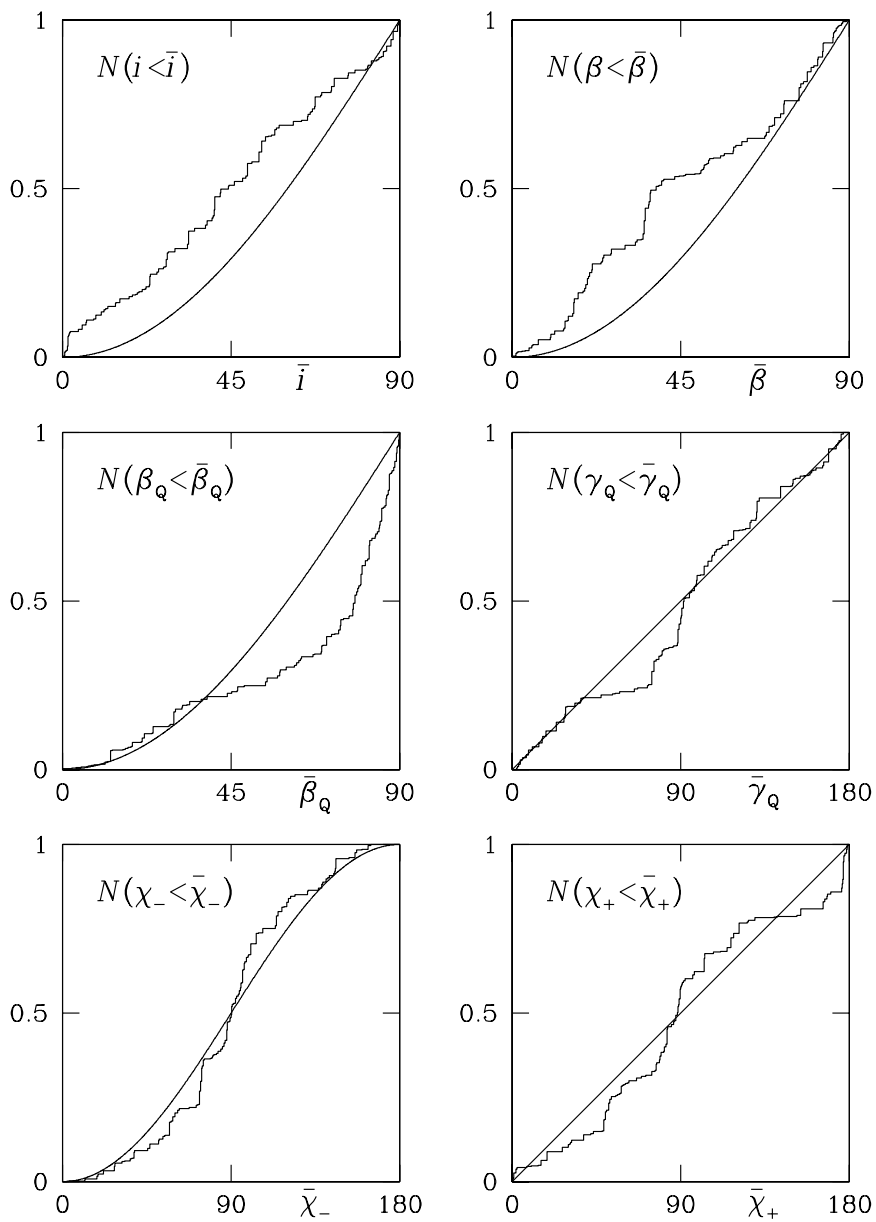

Fig. 6. Cumulative distributions of the angles in Fig. 5, and the corresponding random distributions.

Strictly speaking, the Kolmogorov-Smirnov test in not applicable in our case, because it presupposes a set of "unweighted" measurements of the observational quantity, whereas our determinations are weighted as described in Sect. 2. For a set of $N$ unweighted (different) measurements, all the "steps" of the cumulative distribution are the same $(=1 / N)$. The probability that the distribution of the measurements is consistent with an assigned theoretical distribution obviously depends both on $D$ and on $N$, and, for a given $D$ value, decreases strongly with increasing $N$.

In our case, we are forced to introduce an "effective number" of measurements, $N_{\text {eff }}$. The choice $N_{\text {eff }}=31$ (the number of stars in the sample) would be correct if we had just one model (with the same $\chi^{2}$ value) for each star: as this is not the case, the value $N_{\text {eff }}=31$ is certainly an underestimate. The choice $N_{\mathrm{eff}}=147$ (the overall number of models) would be correct if all the determinations had the same weight: as many models are characterised by rather large $\chi^{2}$ values (hence, rather small weights), $N_{\text {eff }}=147$ is certainly an overestimate. The most reasonable choice is something intermediate: for instance, one could take the quadratic mean of the steps' heights $\Delta_{i}$,

$\langle\Delta\rangle=\left[\frac{1}{n} \sum_{i=1}^{n} \Delta_{i}^{2}\right]^{1 / 2}$,
Table 2. Results of the Kolmogorov-Smirnov test for each angle and for different estimates of the number of measurements, $N_{\text {eff }}$. The $p$ 's are the probabilities (ranging from 0 to 1 ) that the observed and random distributions are consistent. $p_{1}$ corresponds to $N_{\text {eff }}=31$, the number of stars in the sample. $p_{2}$ corresponds to $N_{\text {eff }}=96$ for $i, \beta, \beta_{\mathrm{Q}}, \chi_{-}$; 90 for $\gamma_{\mathrm{Q}} ; 91$ for $\chi_{+}$: these are the nearest-integer approximations to $1 /\langle\Delta\rangle$, where $\langle\Delta\rangle$ is given by Eq. (8). $p_{3}$ corresponds to $N_{\mathrm{eff}}=147$ for $i, \beta, \chi_{-} ; 146$ for $\beta_{\mathrm{Q}} ; 134$ for $\gamma_{\mathrm{Q}} ; 137$ for $\chi_{+}$: these are the numbers of models considered. The reason why this number is less than 147 for $\beta_{\mathrm{Q}}, \gamma_{\mathrm{Q}}$ and $\chi_{+}$is that we ruled out those models where either the "reference" semi-meridian or the point $\mathrm{Q}$ were poorly defined (cf. Fig. 1: very small $\beta, \boldsymbol{u}_{1}$ and $\boldsymbol{u}_{2}$ lying almost in the geographic equator, or nearly parallel to each other).

\begin{tabular}{cclll}
\hline \hline angle & $D$ & \multicolumn{1}{c}{$p_{1}$} & \multicolumn{1}{c}{$p_{2}$} & \multicolumn{1}{c}{$p_{3}$} \\
\hline$i$ & 0.24 & 0.05 & $2 \times 10^{-5}$ & $6 \times 10^{-8}$ \\
$\beta$ & 0.29 & 0.01 & $7 \times 10^{-8}$ & $9 \times 10^{-12}$ \\
$\beta_{\mathrm{Q}}$ & 0.33 & $2 \times 10^{-3}$ & $2 \times 10^{-9}$ & $3 \times 10^{-14}$ \\
$\gamma_{\mathrm{Q}}$ & 0.16 & 0.35 & 0.01 & $1 \times 10^{-3}$ \\
$\chi_{-}$ & 0.12 & 0.69 & 0.09 & 0.02 \\
$\chi_{+}$ & 0.12 & 0.72 & 0.13 & 0.03 \\
\hline
\end{tabular}

where $n$ is the number of models considered, and set $N_{\text {eff }}=$ $1 /\langle\Delta\rangle$.

Table 2 shows the test results for the distributions of the six angles, according to different choices of $N_{\text {eff }}$. It is clear that all the distributions are non-random, and that the largest discrepancies concern $\beta_{\mathrm{Q}}, \beta$, and $i$.

One might suspect that deviations from the random distribution like those of Figs. 5 and 6 could be an artifact due to the inversion algorithm. Because of the large number of parameters and the ensuing complexity of the $\chi^{2}$ hypersurface, noisy observational data might generate some unexpected systematic effect. To check this point we performed extensive numerical simulations, by presenting to the fitting code synthetic and noisy data for a variety of dipole plus quadrupole, randomly oriented models. Such calculations showed that non-random distributions like those of Figs. 5 and 6 can by no means be ascribed to systematic effects of the inversion code.

Let us consider the inclination angle $i$ - the only "nonmagnetic" angle. Its distribution clearly shows an excess of small values. It should be noticed, however, that for most of the stars (22 out of 31 ) the mean field modulus was actually detected: this means that the sample is certainly affected by a selection effect which tends to favour small $i$ values, since positive detections of that quantity require small equatorial velocity, $v_{\mathrm{e}} \sin i$. In fact, the distribution of $i$ for the 9 remaining stars (Fig. 7 left) is very different from that in Fig. 5, and much closer to the random distribution: for $N_{\mathrm{eff}}=9,14$, and 16 (corresponding to the criteria adopted in Table 2 for $p_{1}, p_{2}$, and $p_{3}$, respectively) the probability $p$ resulting from the KolmogorovSmirnov test is $0.91,0.73$, and 0.65 , respectively.

Following another approach, we might consider the distribution of $i$ for all stars with sufficiently small rotational velocity (period $P>P_{0}$ ): such sample should likely be unaffected by selection effects. Setting $P_{0} \simeq 40$ days - actually, any number of days between 35 and 48 - we get a sub-sample of 10 stars (none 

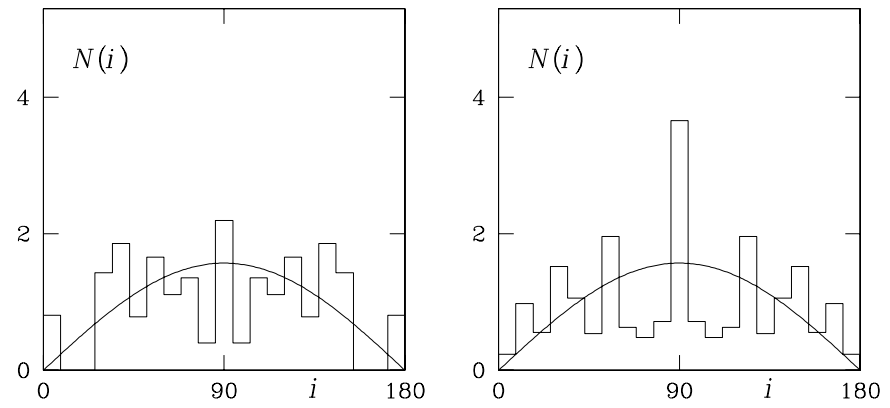

Fig. 7. Distribution of the inclination angle $i$ for the 9 stars with no $\langle\mathcal{B}\rangle$ observations (left), and for the 10 stars with rotational period larger than 40 days (right).

of which pertaining to the former 9-star sample) leading to the distribution of Fig. 7, right. For $N_{\mathrm{eff}}=10,30$, and 37, corresponding to the same criteria as above, we have $p=0.82,0.21$, and 0.12 , respectively. This probability decreases strongly with decreasing $P_{0}$.

On the whole, the difference from the random distribution looks rather reduced, though some excess of small $i$ values remains. This is not fully understood. It could be due either to inadequacy of the dipole plus quadrupole model, or to a weak, but real intrinsic non-randomness of the star sample, although it should be noted that this latter phenomenon does not have an obvious physical justification. We recall that - contrary to axisymmetric models - our magnetic model involves no degeneracy between the angles $i$ and $\beta$, the only "physical" degeneracy being that of Eq. (2). Thus a randomization - so to say - of the $i$ distribution at expense of the $\beta$ distribution is not allowed in our case (see Landstreet \& Mathys 2000).

Let us now turn our attention to the "magnetic" parameters of the model. Since the magnetic structure may plausibly be connected with the star's rotation, we looked for possible correlations between the magnetic parameters and the rotational period $P$.

Figure 8 shows the dipole amplitude $B_{\mathrm{d}}$ (upper panel) and the ratio of quadrupole to dipole amplitude $B_{\mathrm{q}} / B_{\mathrm{d}}$ (lower panel) as functions of $P$. The figure refers to all the 147 models associated with the 31 stars of our sample. Both quantities, and particularly the latter, decrease on average with increasing $P$ : faster rotators seem to be characterised by stronger and predominantly quadrupolar fields.

Similarly, Fig. 9 shows the phase-averaged mean field modulus as a function of $P$. The number of dots is much smaller than in Fig. 8 because - as already noted - the different models of a given star yield almost the same $\overline{\langle\mathcal{B}\rangle}$ value. Consistently with the data in Fig. $8, \overline{\langle\mathcal{B}\rangle}$ tends to decrease with increasing $P$.

As far as the magnetic geometry is concerned, consider first the quadrupolar component. The first step is to find the location of the point $\mathrm{Q}$ on the stellar surface (cf. Fig. 1). The plot in Fig. 10 shows the location of $\mathrm{Q}$ in the $\left(\beta_{\mathrm{Q}}, \gamma_{\mathrm{Q}}\right)$ plane for all models ${ }^{2}$. It turns out that the dots tend to cluster round $\beta_{\mathrm{Q}} \simeq 90^{\circ}$, and, to a smaller extent, round $\gamma_{\mathrm{Q}} \simeq 0^{\circ}$ and $\gamma_{\mathrm{Q}} \simeq 90^{\circ}$ : such characteristics were already apparent from the histograms

\footnotetext{
${ }^{2}$ Owing to the symmetry properties of Eqs. (2) and (3), the plot can be restricted to $1 / 4$ of the whole spherical surface.
}
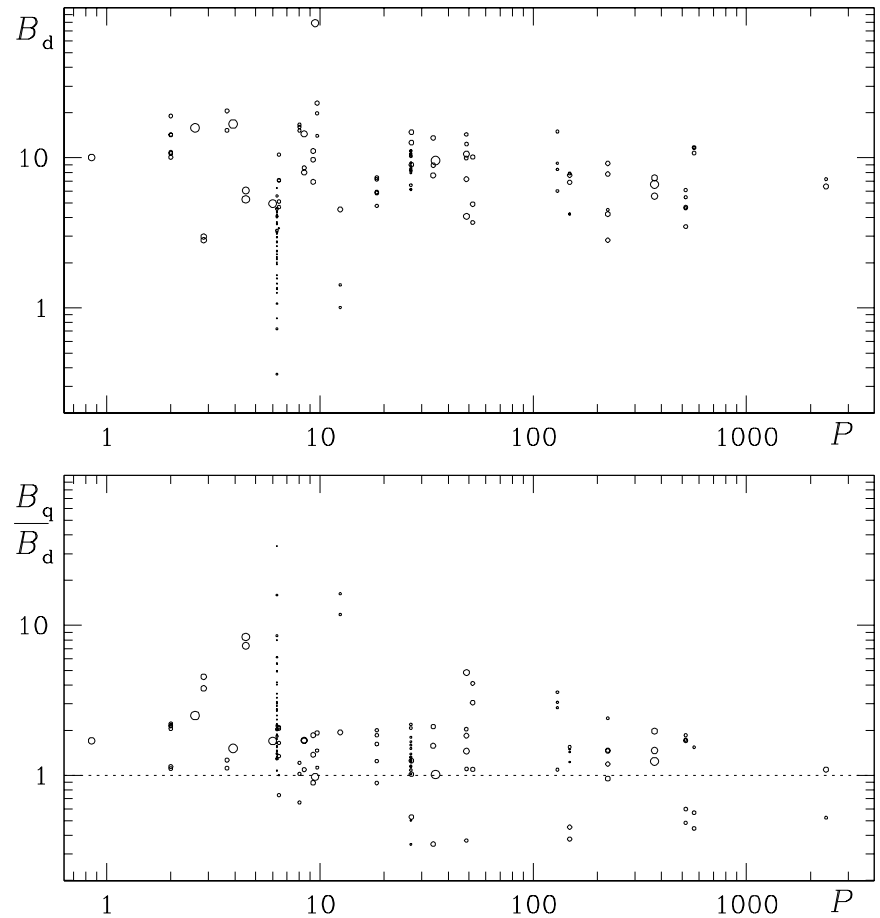

Fig. 8. Dipole amplitude (upper panel) and ratio of quadrupole to dipole amplitude (lower panel) versus rotational period. $P$ is expressed in days, $B_{\mathrm{d}}$ in $\mathrm{kG}$. The dots' area is proportional to the weight $\rho_{j k}$ of Eq. (6).

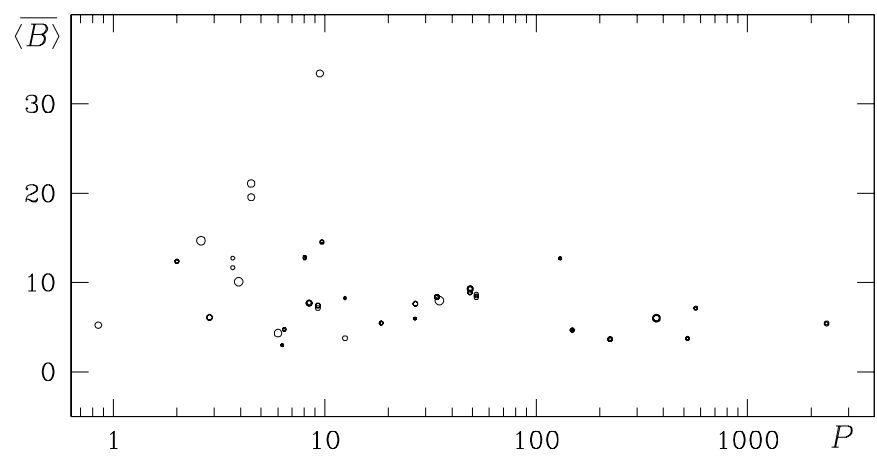

Fig. 9. The phase-averaged mean field modulus (in $\mathrm{kG}$ ) against the rotational period (in days).

of Fig. 5. But the most interesting aspect arises when connecting the dots corresponding to all the models of a given star: one finds that, for a number of stars, the location of the point $\mathrm{Q}$ lies within a small region around the point $\mathrm{A} \equiv\left(90^{\circ}, 90^{\circ}\right)$. There are seven stars of this kind, shown in the lower panel of Fig. 10. For three of them - HD 96446, HD 119419, and HD 147010, labelled in the figure as No. 14, 17, and 24, respectively - the location of the point $\mathrm{Q}$ is univocal (one minimum in the $\chi^{2}$ hypersurface), while for the others - HD 70331, HD 137509, HD 208217 and HD 318107 (labelled in the figure with 8, 20,31, and 33, respectively) - there are several possible locations. In any case, for all of them the point $\mathrm{Q}$ lies within a small spherical cap, centred at the point A and having a width $d \simeq 22^{\circ}$ (which amounts to about $7 \%$ of the spherical surface) - see Fig. 11. These stars will be referred to in the following as "class I". 

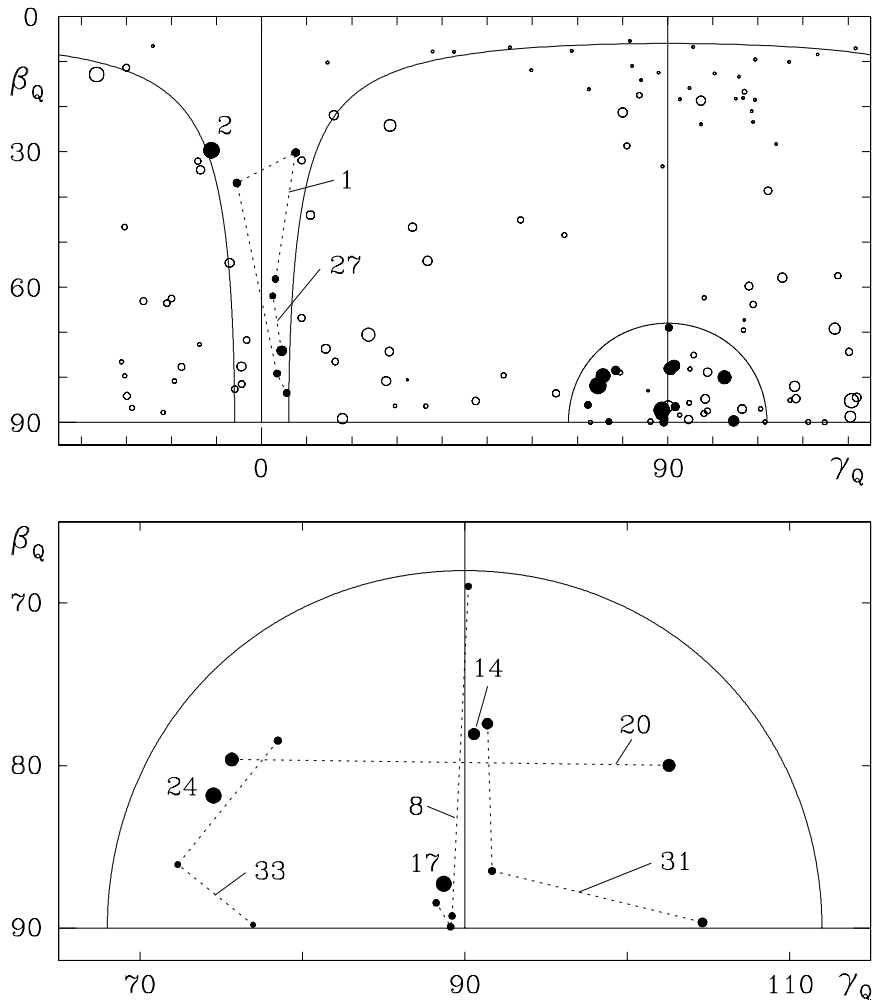

Fig. 10. Upper panel: the position of the point $\mathrm{Q}$ in the $\left(\beta_{\mathrm{Q}}, \gamma_{\mathrm{Q}}\right)$ plane for each of the fitting models. Angles are expressed in degrees. The $\gamma_{\mathrm{Q}}$ axis spans a $180^{\circ}$ interval centred at $45^{\circ}$ in order to show clearly the regions of interest (cf. Fig. 11): two spherical caps with widths $d=22^{\circ}$ and $d=84^{\circ}$ centred at the point $\mathrm{A} \equiv\left(90^{\circ}, 90^{\circ}\right)$. The dots' area is proportional to $\rho_{j k}$ defined in Eq. (6). Full dots indicate "class I" and "class II" stars, labelled according to their reference number in Table 1. Class II stars HD 2453, HD 12288, and HD 187474 (labelled as 1,2, and 27) have five, one, and two models, respectively. Lower panel: an enlargement of the class I region, showing the connections between models of the same star. Class I stars are HD 70331, HD 96446, HD 119419, HD 137509, HD 147010, HD 208217, and HD 318107 (indicated in the figure as No. 8, 14, 17, 20, 24, 31, and 33).

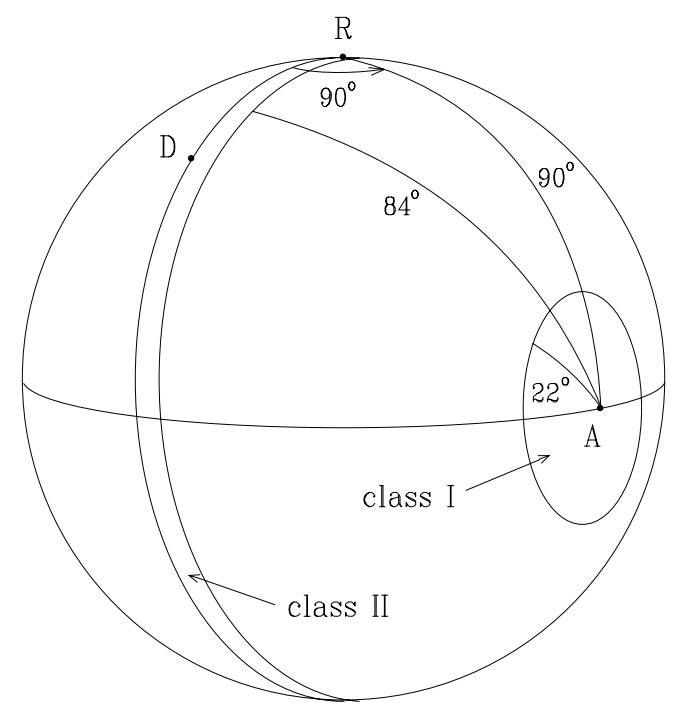

Fig. 11. Location of the quadrupole pole Q on the stellar surface for "class I" and "class II" stars.

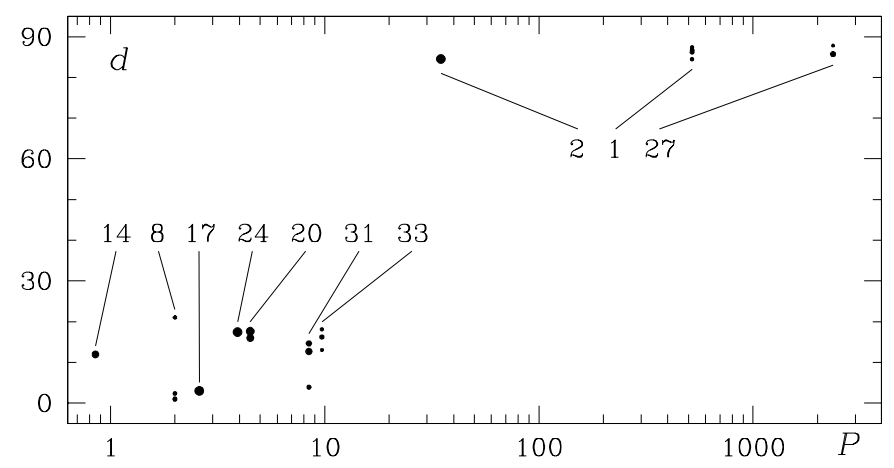

Fig. 12. The angular distance $d$ (in degrees) of the point $\mathrm{Q}$ from the point $\mathrm{A} \equiv\left(90^{\circ}, 90^{\circ}\right)$ as a function of the rotational period (in days), for class I (lower left) and class II (upper right) stars. Dots' area is proportional to $\rho_{j k}$ of Eq. (6).

Similarly, we found that for 3 other stars - HD 2453, HD 12288, and HD 187474 (No. 1, 2, and 27, respectively) - the point Q lies within a small "ring" between $d=84^{\circ}$ and $d=90^{\circ}$, i.e., a $6^{\circ}$ ring around the meridian containing the stellar rotation axis and the dipole axis (about $10 \%$ of the spherical surface). These stars, denoted as "class II" in the following, are shown in the upper panel of Fig. 10.

For the remaining stars, the location of the point $\mathrm{Q}$ is usually scattered throughout the $\left(\beta_{\mathrm{Q}}, \gamma_{\mathrm{Q}}\right)$ plane according to the different models, so that no precise characterisation is possible.

It should be noticed that, in terms of the angles $\beta_{1}, \beta_{2}, \gamma_{1}$, $\gamma_{2}$, class I stars are characterised by the property $\gamma_{1}=0^{\circ}$ or $180^{\circ}$ and $\gamma_{2}=0^{\circ}$ or $180^{\circ}$ (the unit vectors $\boldsymbol{u}_{1}$ and $\boldsymbol{u}_{2}$ lie in the plane of the rotation axis and dipole axis). It follows that the degeneracy of Eq. (2) does not apply to such stars: this can be formally seen by combining Eqs. (2) and (4).

Let us now examine whether any correlation exists between the location of the point $\mathrm{Q}$ on the stellar surface and the rotational period. If we restrict attention to class I and class II stars (for which such location is more precise), we obtain the remarkable result shown in Fig. 12: there is striking evidence that class I stars are short-period, and class II stars are longperiod. A similar result was anticipated by Bagnulo (2001).

The idea of a general "law", valid for all the stars in our sample, according to which the angular distance $d$ of the point $\mathrm{Q}$ from the point $\mathrm{A} \equiv\left(90^{\circ}, 90^{\circ}\right)$ is an increasing function of the rotational period, looks really tempting. However, we just observed that for a star other than class I or II, the locations of the point $\mathrm{Q}$ associated with the various models are generally far apart in the $\left(\beta_{\mathrm{Q}}, \gamma_{\mathrm{Q}}\right)$ plane - which entails scattered values (i.e., both large and small) for $d$. In fact, when $d$ is plotted against $P$ for all models of all the stars in the sample, we obtain the results of Fig. 13, which look rather ambiguous. In particular, some long-period stars (especially HD 116458 , HD 188041, HD 51684, and HD 93507 - No. 16, 28, 5, and 12 , respectively) have both large- $d$ and small- $d$ models. (Note that in the case of HD 93507, the determination of the magnetic observables - hence the modelling - is hampered by the fact that the distribution of Fe over the stellar surface is rather strongly inhomogeneous.) 


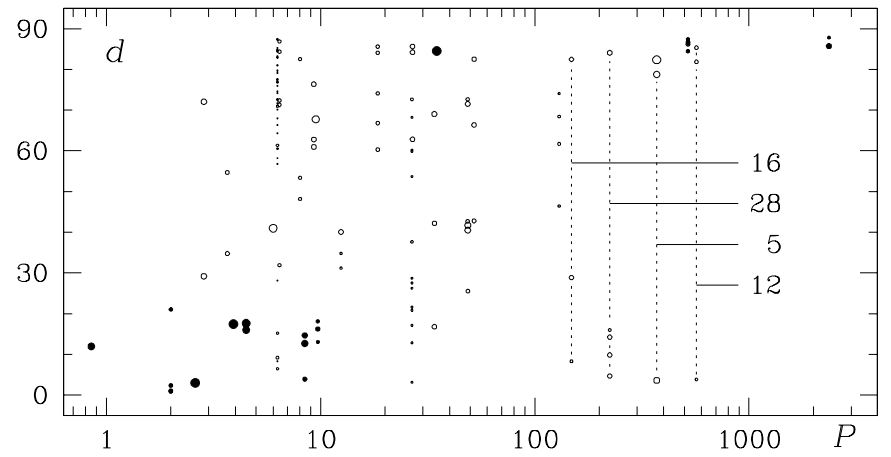

Fig. 13. Same as Fig. 12 for all models of all the stars in the sample. The vertical lines connect the dots corresponding to the different models of stars HD 116458, HD 188041, HD 51684, and HD 93507, that are labelled in the figure as $16,28,5$, and 12 , respectively.

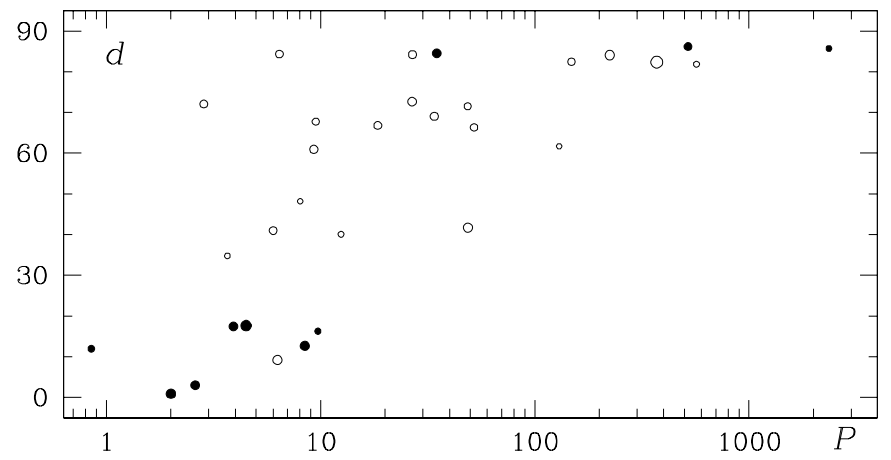

Fig. 14. Same as Fig. 13 for the best models of all the stars in the sample. Full dots are class I and class II stars. Dots' area is proportional to the weight $\rho_{j}$ of Eq. (5), which is more appropriate in this context.

However, if we restrict attention to the best model (associated with the absolute $\chi^{2}$ minimum) of each of the 31 stars, we obtain the plot in Fig. 14. It really seems that the data of the whole sample support (approximately) the "law" mentioned above.

Finally, one could suspect that a relationship exists between $d$ and $\overline{\langle\mathcal{B}\rangle}$, since both quantities are somewhat related to the rotation period. However, inspection to the plot of $d$ vs. $\overline{\langle\mathcal{B}\rangle}$ did not reveal any obvious correlation.

In order to characterise the quadrupole orientation, one needs to know - beside the location of the point $\mathrm{Q}-$ the directions of the unit vectors $\boldsymbol{u}_{1}$ and $\boldsymbol{u}_{2}$ in their plane, i.e., the angles $\chi_{1}$ and $\chi_{2}$ or, equivalently, $\chi_{-}$and $\chi_{+}$. Contrary to $\mathrm{Q}$, those directions proved to be basically uncorrelated with the rotational period: this is obvious even if we restrict the analysis to the best models, as shown in Fig. 15. The only "anomalies" of $\chi_{-}$and $\chi_{+}$are those appearing in Fig. 5: some excess of $\chi_{-}$values around $90^{\circ}$, and of $\chi_{+}$values around $0^{\circ}$ and $90^{\circ}$. Though the discrepancies from the relevant random distributions are less marked than for the other angles (cf. Table 2), it is interesting to observe that the $\chi_{\text {- distribution tends to rule }}$ out linear quadrupole models $\left(\boldsymbol{u}_{1}\right.$ parallel or antiparallel to $\boldsymbol{u}_{2}$, which entails $\chi_{-}=0^{\circ}$ or $\left.180^{\circ}\right)$.

Next we consider the dipole orientation. Figure 16 shows the angle $\beta$ as a function of the rotational period. The upper panel refers to all models of all the 31 stars, and shows
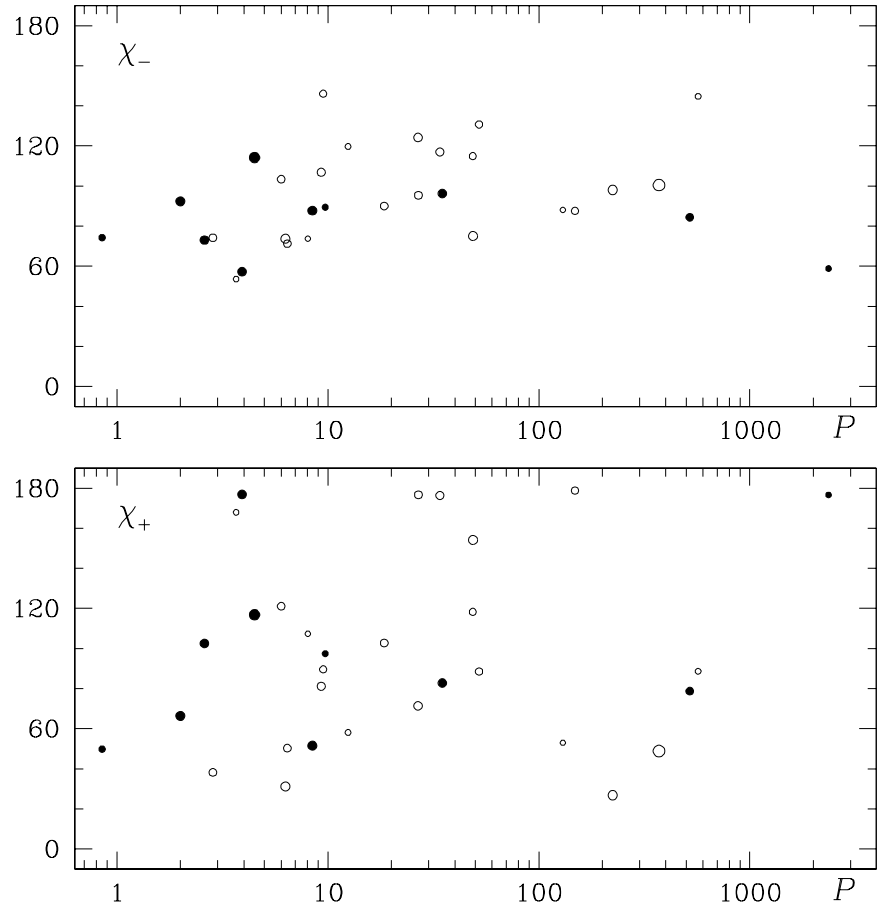

Fig. 15. The angles $\chi_{-}$(upper panel) and $\chi_{+}$(lower panel) - expressed in degrees - against the rotational period in days. Only the best-fit values are shown. Full/open dots and dots' area have the same meaning as in Fig. 14.

a deficiency of large $\beta$ values for long period stars. If we confine attention to the best-fit models (lower panel), we better see that $\beta$ tends to decrease with increasing rotational period. This is in agreement with the main result of Landstreet \& Mathys (2000): $\beta$ is usually small for slow rotators and large for fast rotators. Such agreement is especially significant for long-period stars, where the quadrupolar component is less important (see Fig. 8, lower panel), so that the orientation of the magnetic structure is dominated by the dipole orientation. A notable exception to this general trend is represented by HD 187474. This star has a rotation period of $2364 \mathrm{~d}$ (the longest in the $g$ sample), and the best-model predicts a tilt angle between dipole axis and rotation axis of $80^{\circ}$. A detailed study of its chemical peculiarity has been recently presented by Strasser et al. (2001).

On the whole, the results above show the existence of some correlation between the magnetic structure of the stars of our sample and the rotational period.

\section{Analysis based on 3 magnetic observables}

As recalled at the end of Sect. 2 , the consistency of $\left\langle\mathcal{B}^{2}+\mathcal{B}_{z}^{2}\right\rangle^{1 / 2}$ and $\langle\mathcal{B}\rangle$ observations is not completely ascertained. For this reason we repeated the analysis of Sect. 3 by neglecting either kind of measurements. However, such "partial" analyses imply that some stars "go out" of the sample, so that the "global" sample of Sect. 3 and the two partial samples are not directly comparable.

In fact, in Paper III we determined the minimum combinations of magnetic observables which allow a dipole plus (non linear) quadrupole configuration to be recovered. In particular, 

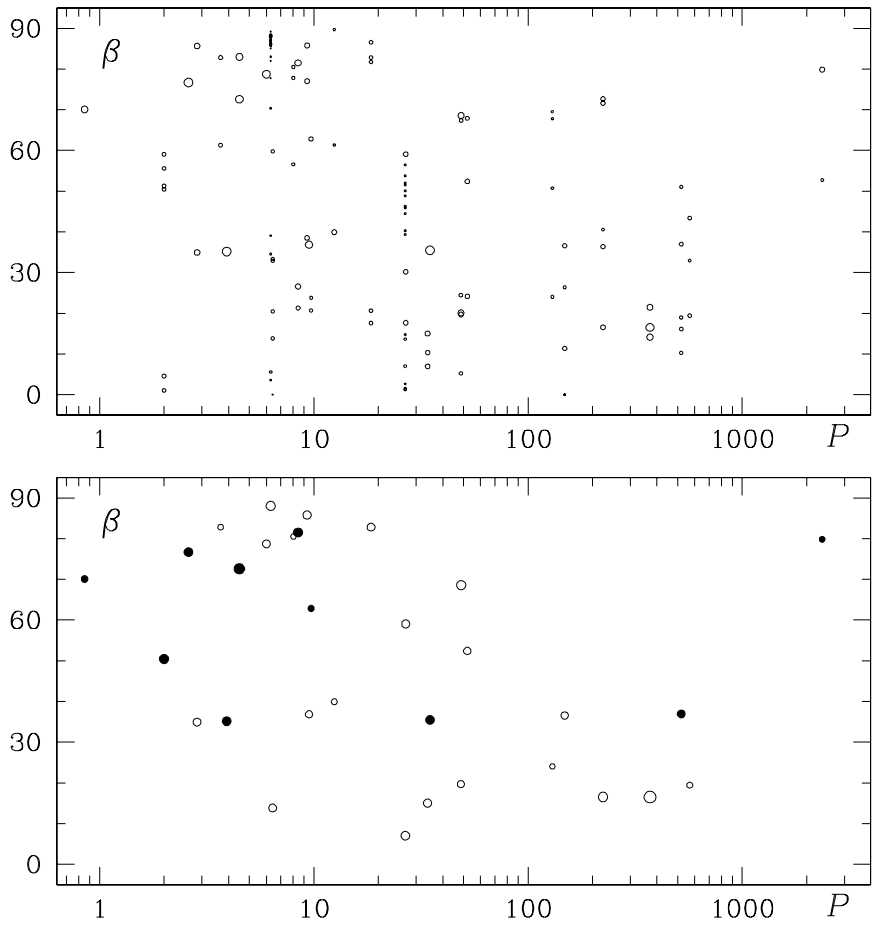

Fig. 16. The angle $\beta$ (in degrees) versus the rotational period (in days), for all models (upper panel) and for best-fit models (lower panel). Dot's area is proportional to $\rho_{j k}$ and to $\rho_{j}$ in the upper and lower panel, respectively. Full dots are class I and class II stars.

Table 1 of that paper shows that the configuration can be recovered from (a sufficient number of) $\left\langle\mathcal{B}_{z}\right\rangle$ and $\left\langle\mathcal{B}^{2}+\mathcal{B}_{z}^{2}\right\rangle^{1 / 2}$ observations, as well as from $\left\langle\mathcal{B}_{z}\right\rangle$ and $\langle\mathcal{B}\rangle$ observations; whereas it cannot be recovered either from $\left\langle\mathcal{B}_{z}\right\rangle$ alone, or from $\left\langle\mathcal{B}_{z}\right\rangle$ and $v_{\mathrm{e}} \sin i\left\langle d \mathcal{B}_{z}\right\rangle$. Bearing in mind these results, we see that the subsamples for our partial analyses contain 25 stars each.

These are shown in the last two columns of Table 1, where the label " $p 1$ " means that the fit is based on all available observations except $\left\langle\mathcal{B}^{2}+\mathcal{B}_{z}^{2}\right\rangle^{1 / 2}$, while the label " $p 2$ " means that it is based on all available observations except $\langle\mathcal{B}\rangle$. It should be noticed that stars HD 75445 and HD 335238, which in principle pertain to sub-sample $p 2$ as for both of them there are measurements of $\left\langle\mathcal{B}_{z}\right\rangle, v_{\mathrm{e}} \sin i\left\langle d \mathcal{B}_{z}\right\rangle$, and $\left\langle\mathcal{B}^{2}+\mathcal{B}_{z}^{2}\right\rangle^{1 / 2}$, are actually excluded from $p 2$ because of the small number of observations ( 9 in all), which renders the fit impossible. On the other hand, stars HD 61468, HD 94660, and HD 142070, which in the "global" investigation give rise to low-quality fits $\left(\chi_{\min }^{2}>8\right)$ so that they were excluded from the statistical analysis, in the "partial" investigations give rise to much better fits, thus they have been included in the analysis.

If we restricted the study to the stars common to all three samples $g, p 1$, and $p 2$, we would be left with only 13 objects too small a number for a statistical analysis. On the other hand, a direct comparison of samples $g$ and $p 1$, or $g$ and $p 2$, is not very meaningful because the fit of several of the common stars is identical by definition: for instance, this is the case for the $g$-fit and the $p 1$-fit of star HD 12288, because for that star there are no observations of $\left\langle\mathcal{B}^{2}+\mathcal{B}_{z}^{2}\right\rangle^{1 / 2}$, hence either fit is in fact the same fit (based on $\left\langle\mathcal{B}_{z}\right\rangle$ and $\langle\mathcal{B}\rangle$ observations).
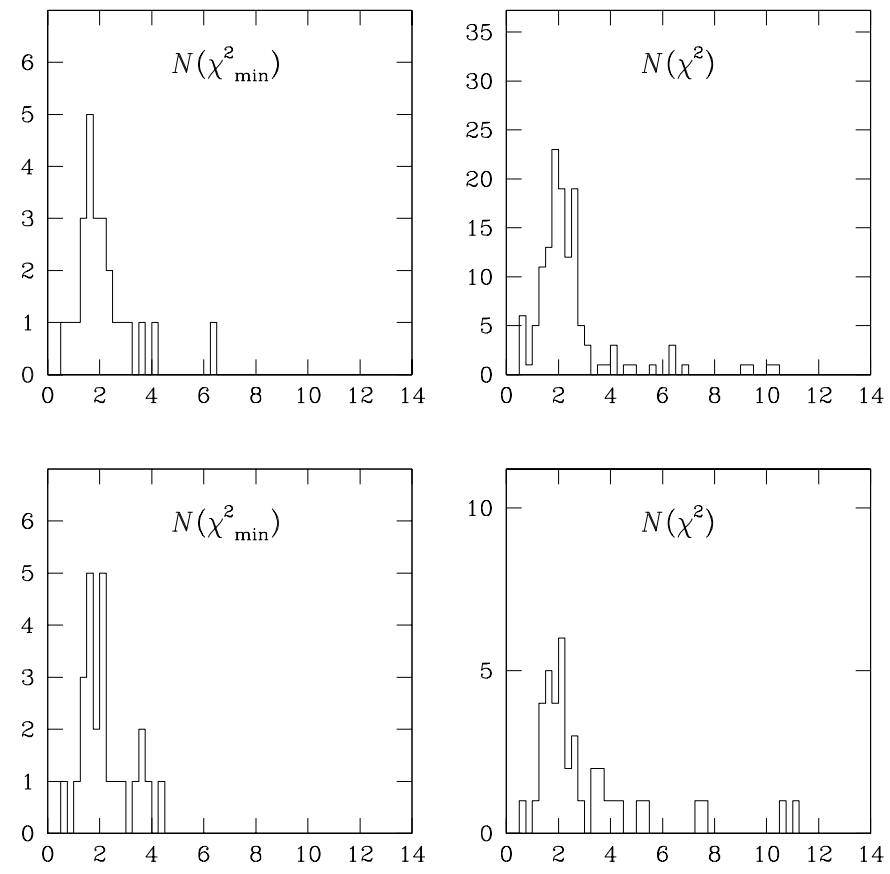

Fig. 17. Same as Fig. 2 for the stars of samples $p 1$ (upper panels) and $p 2$ (lower panels). Either sample contains 25 stars.

For these reasons, the correct attitude is to forget comparisons and to look at the "global" and "partial" analyses as separate investigations. The final goal is to see whether the overall picture of magnetic $\mathrm{CP}$ stars arising from analyses of different kind (in the sense specified above) is the same or not; where by "overall picture" we intend the presence of recurrent features and/or correlations among the parameters of the magnetic model.

Columns $p 1$ and $p 2$ of Table 1 show the value of the reduced $\chi^{2}$ for the best-fit model of the 25 stars pertaining to either sample. Such data is also shown in the left panels of Fig. 17. The two distributions are similar to that of the $g$-case (cf. Fig. 2 left), though somewhat displaced towards smaller values - which reflects the difficulties encountered in fitting together $\left\langle\mathcal{B}^{2}+\mathcal{B}_{z}^{2}\right\rangle^{1 / 2}$ and $\langle\mathcal{B}\rangle$ observations. In the right panels of Fig. 17 we show the distributions of the reduced $\chi^{2}$ for all models (absolute and relative $\chi^{2}$ minima): on the whole, there are 140 models for the $p 1$-case $\left(7\right.$ of which with $\chi^{2}>14$ ) and 60 models for the $p 2$-case (20 of which with $\chi^{2}>14$ ). These figures show that the average number of models per star - i.e., the number of minima in the $\chi^{2}$ hypersurface - increases strongly when $\langle\mathcal{B}\rangle$ observations are included: from 2.4 for the $p 2$-case to 4.9 for the $g$-case and 5.6 for the $p 1$-case. Thus inclusion of $\langle\mathcal{B}\rangle$ observations renders the $\chi^{2}$ hypersurface much more complex: this is possibly due to a stronger degeneracy of $\langle\mathcal{B}\rangle$ compared to the other magnetic observables (different dipole plus quadrupole configurations leading to similar $\langle\mathcal{B}\rangle$ curves). As in Sect. 3, we restricted the statistical analysis to the models with $\chi^{2}<8$.

Figure 18 shows the distributions of the dipole amplitude, and of the ratio between quadrupole and dipole amplitude, for the two "partial" samples, while Fig. 19 refers to the phaseaveraged mean field modulus. Such distributions are in fact 

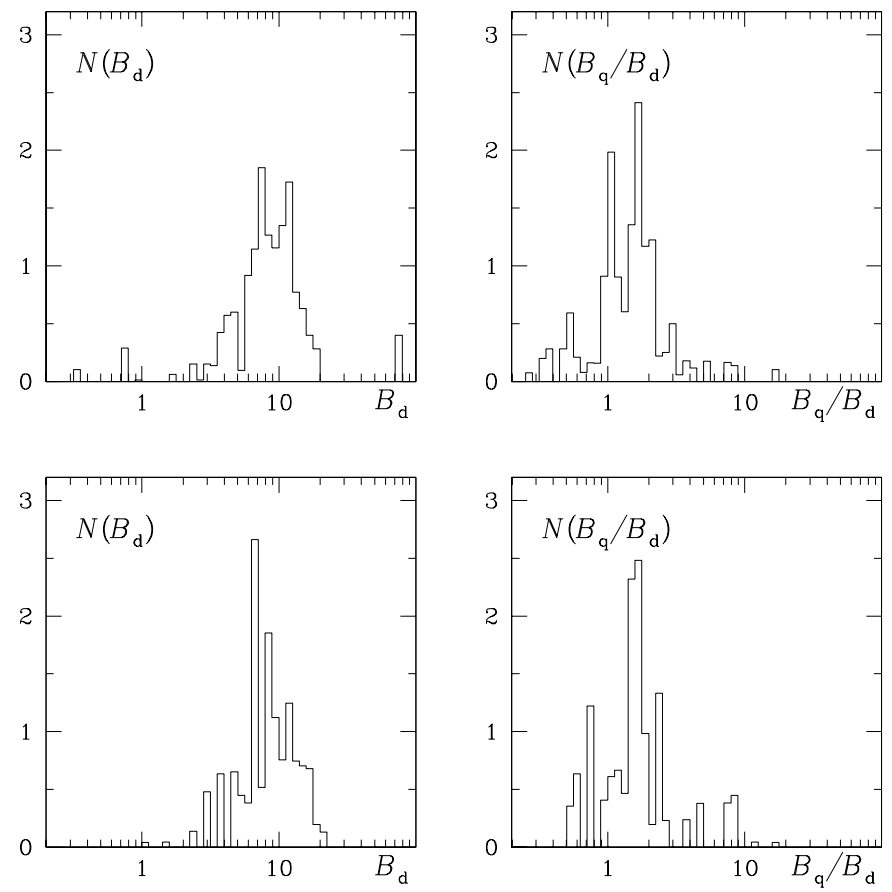

Fig. 18. Same as Fig. 3 for samples $p 1$ (upper panels) and $p 2$ (lower panels).

quite similar to those of Sect. 3 (cf. Figs. 3 and 4). Note that Babcock's star does not belong to the $p 2$-sample (see Table 1).

It is interesting to compare our Figs. 4 and 19 with the histogram showing the observed phase-averaged magnetic field modulus presented in Fig. 47 of Mathys et al. (1997). We recall that Fig. 4 is based on the modelling results of all available magnetic observables; the left panel of Fig. 19 is obtained from the results of the analysis of all quantities except the mean quadratic field; the right panel of Fig. 19 from results of the analysis of all quantities except the mean field modulus; Fig. 47 of Mathys et al. (1997) is purely based on the observations, and includes stars that have not been monitored throughout the rotation cycle. A similar overall picture emerges from all these figures. A certain degree of consistency is to be expected for the following reason: the mean field modulus is generally a quantity that does not change much over the rotation phase, and even few observed points, or a very rough model are sufficient to characterise the $\overline{\langle\mathcal{B}\rangle}$ curve with good approximation. We note in particular that all figures show a definite lower limit in the $\overline{\langle\mathcal{B}\rangle}$ distribution at about $3 \mathrm{kG}$ - this characteristic has been already discussed in Mathys et al. (1997), and in Hubrig et al. (2000). It is interesting to note that the same result is here recovered also for stars with no magnetically split lines, though still with relatively low $v_{\mathrm{e}} \sin i$ values. Notably the same sharp cut-off is found from the modelling of $\left\langle\mathcal{B}_{z}\right\rangle, v_{\mathrm{e}} \sin i\left\langle d \mathcal{B}_{z}\right\rangle$, and $\left\langle\mathcal{B}^{2}+\mathcal{B}_{z}^{2}\right\rangle^{1 / 2}$ (see right panel of Fig. 19).

The distributions of the angles $i, \beta, \beta_{\mathrm{Q}}, \gamma_{\mathrm{Q}}, \chi_{-}, \chi_{+}$for the $p 1$ and $p 2$ cases are shown in Figs. 20 and 21, respectively. It appears that the deviations from the relevant random distributions are basically the same as in the $g$-case (cf. Fig. 5). These include an excess of small $\beta$ values and large $\beta_{\mathrm{Q}}$ values, and, to

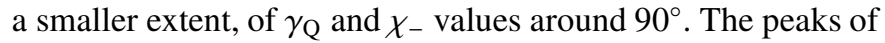
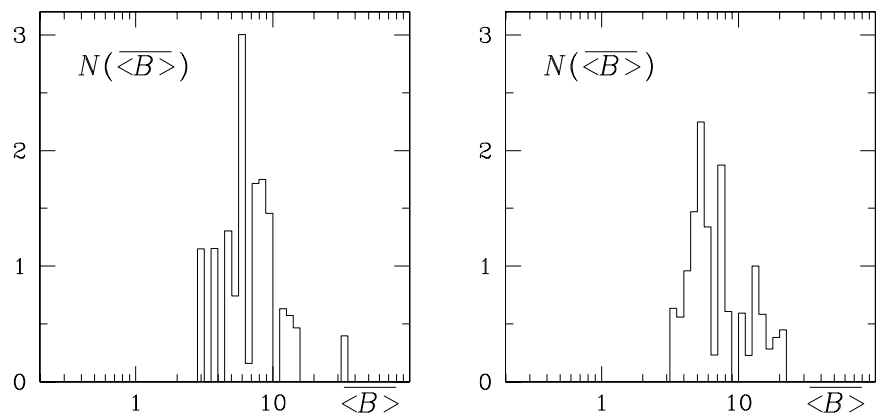

Fig. 19. Same as Fig. 4 for $p 1$ (left) and $p 2$ (right).
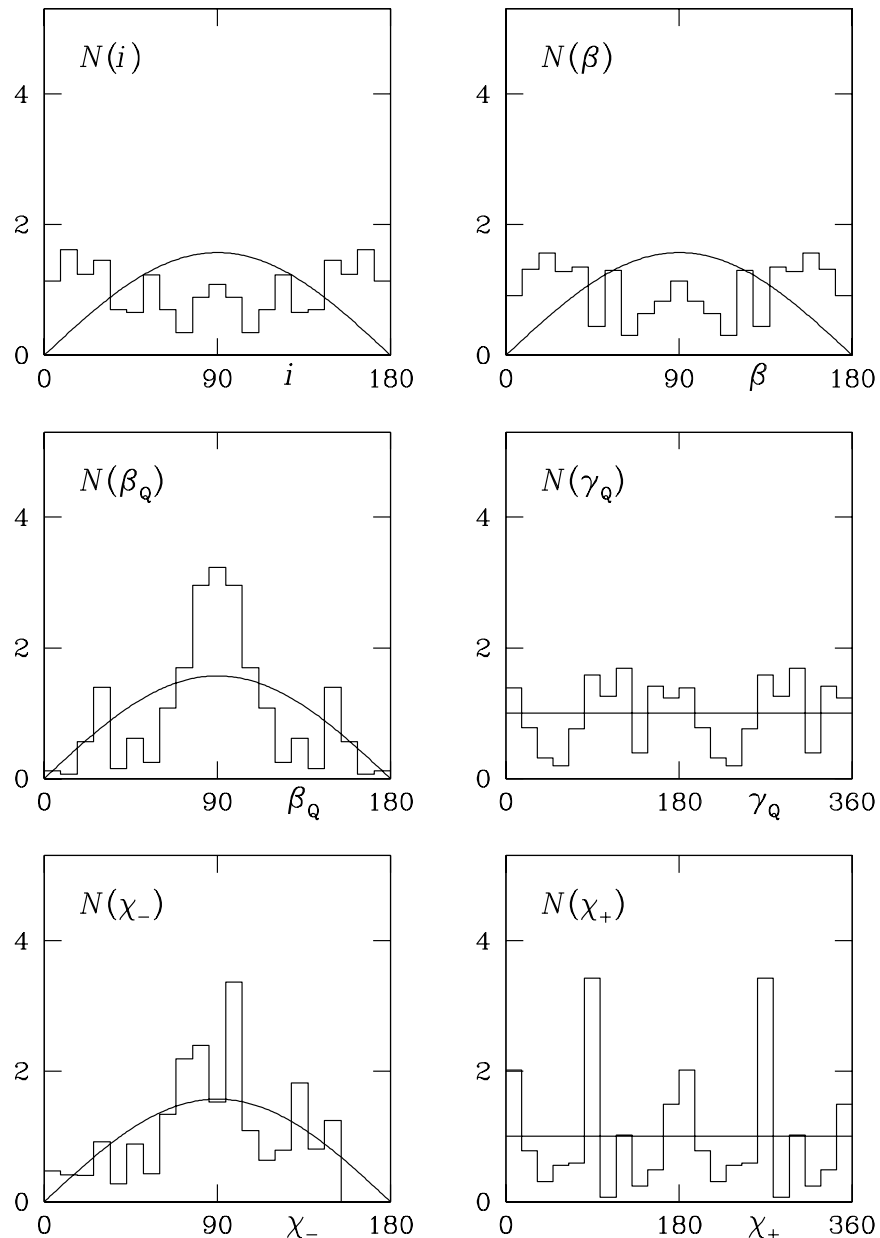

Fig. 20. Same as Fig. 5 for the $p 1$-case.

the "barycentric angle" $\chi_{+}$around $0^{\circ}$ and $90^{\circ}$ are still visible, though the latter is somewhat shifted in the $p 2$-case.

As far as the inclination angle $i$ is concerned, it should be borne in mind that - contrary to the $g$-case $-\langle\mathcal{B}\rangle$ was detected for all stars of the $p 1$-sample. The selection effect is therefore larger than in the $g$-case, so that the excess of small $i$ values is even more prominent (see Fig. 20). On the other hand, the p2-sample contains a larger percentage of long-period stars (say, with $P>40$ days) with respect to the $g$-sample, which explains why the excess of small $i$ values is less prominent (see Fig. 21). 

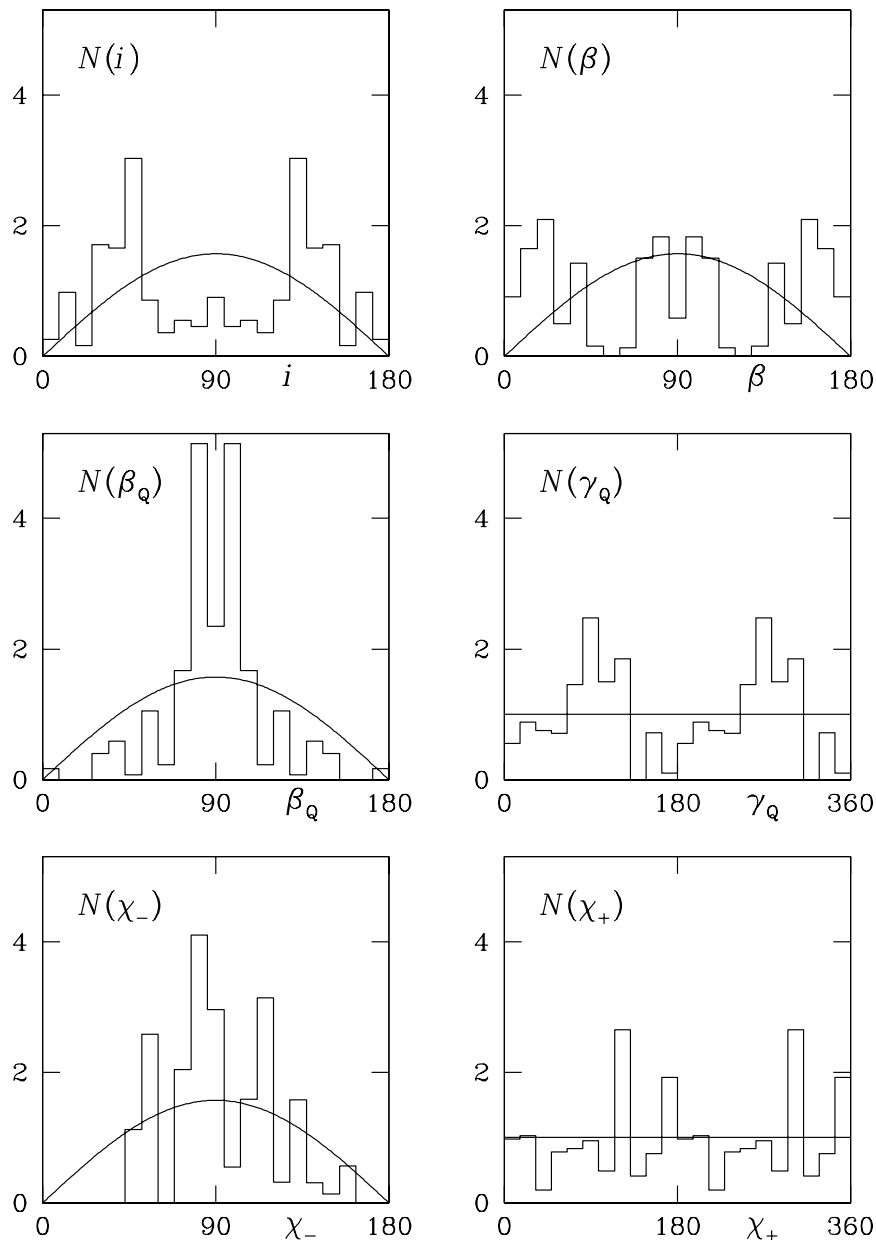

Fig. 21. Same as Fig. 5 for the $p 2$-case.

Consider next the correlations with the rotational period. Concerning the field strength, the behaviour of the dipole and quadrupole amplitudes is quite similar to the $g$-case; we show only the phase-averaged mean field modulus (Fig. 22), which tends to decrease with increasing $P$ as in Fig. 9.

As to the quadrupole orientation, we repeated for the $p 1$ and $p 2$ cases the same analysis on the location of the point $\mathrm{Q}$ on the spherical surface described in Sect. 3 (see Fig. 10). For both cases, we found a number of "class I" and "class II" stars (cf. Fig. 11), and we plotted the angular distance $d$ between points $\mathrm{Q}$ and A against the rotational period (cf. Figs. 12 to 14). The final results (based on the best fit of each star) are shown in Fig. 23.

Altogether, the $p 1$-case yields a picture similar to the $g$-case (see Fig. 14), with the exception of star HD 94660 (absent in the $g$-sample) - which is the longest-period star of all the $34 \mathrm{ob}$ jects and which is characterised by a small $d$ value - and possibly of HD 116458.

By contrast, the $p 2$-case yields a very different picture, which tends to deny the existence of a correlation between $d$ and $P$. In particular, it is worth noticing the behaviour of stars HD 116458, HD 93507, and HD 51684. As shown in Fig. 13, in the $g$-case they have both large- $d$ and small- $d$ models, but the best-fit ones are the former (see Fig. 14). In the $p 2$-case, on the contrary, the best-fit models of all three stars correspond to
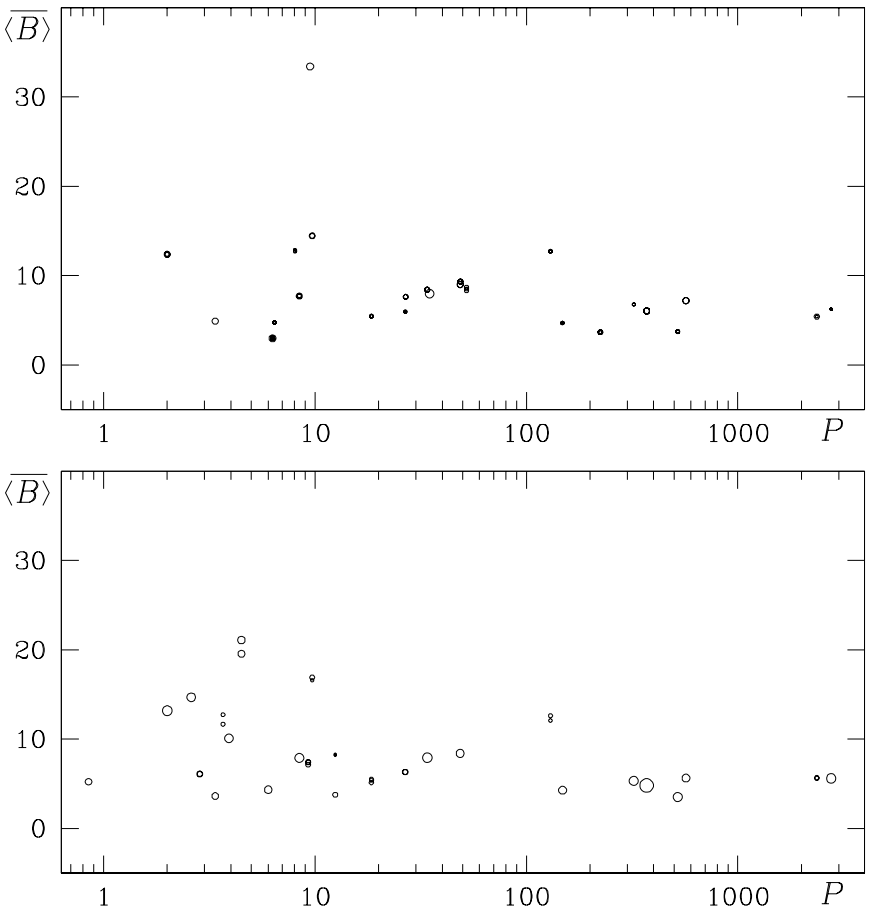

Fig. 22. Same as Fig. 9 for cases $p 1$ (upper panel) and $p 2$ (lower panel). The larger dots' area in the lower panel is just a consequence of the smaller average number of models per star in the $p 2$-case.
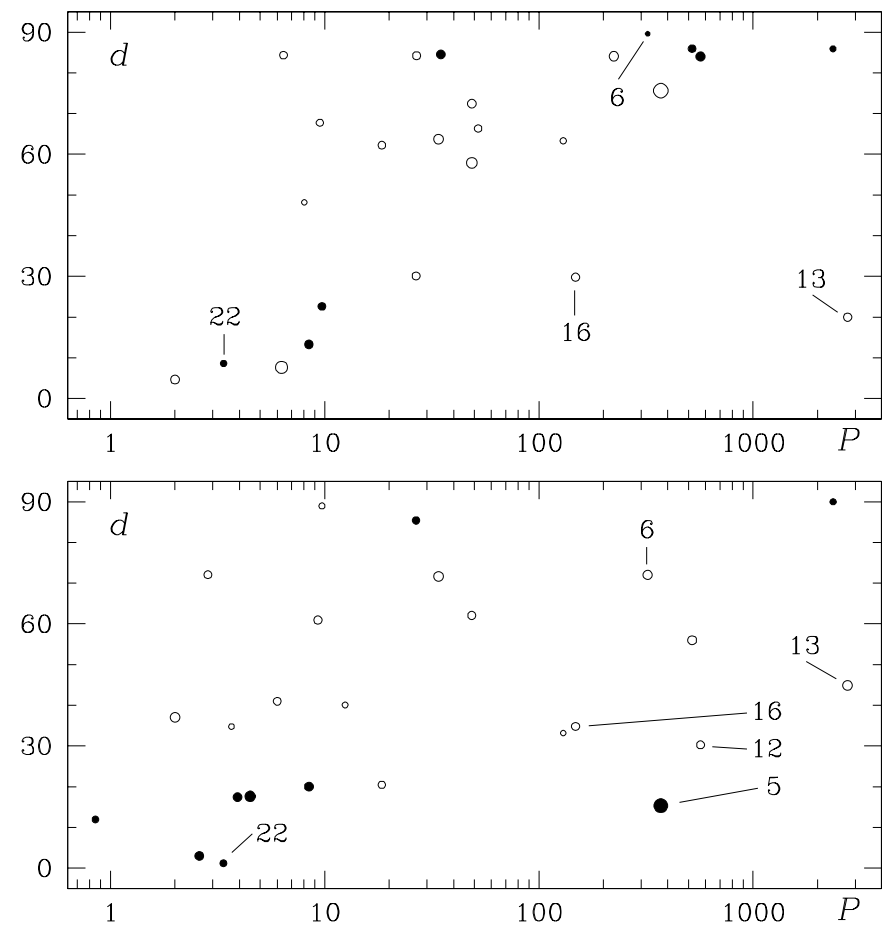

Fig. 23. Same as Fig. 14 for cases $p 1$ (upper panel) and $p 2$ (lower panel). Full dots represent class I and class II stars, defined as in Sect. 3. Some stars are labelled by their reference number of Table 1.

small $d$ values. On the other side, it should also be noticed that the short-period star HD 142070 (absent in the $g$-case) proves to be a class I object both in the $p 1$ and the $p 2$ case, and that the long-period star HD 61468 (also absent in the $g$-case) proves to 

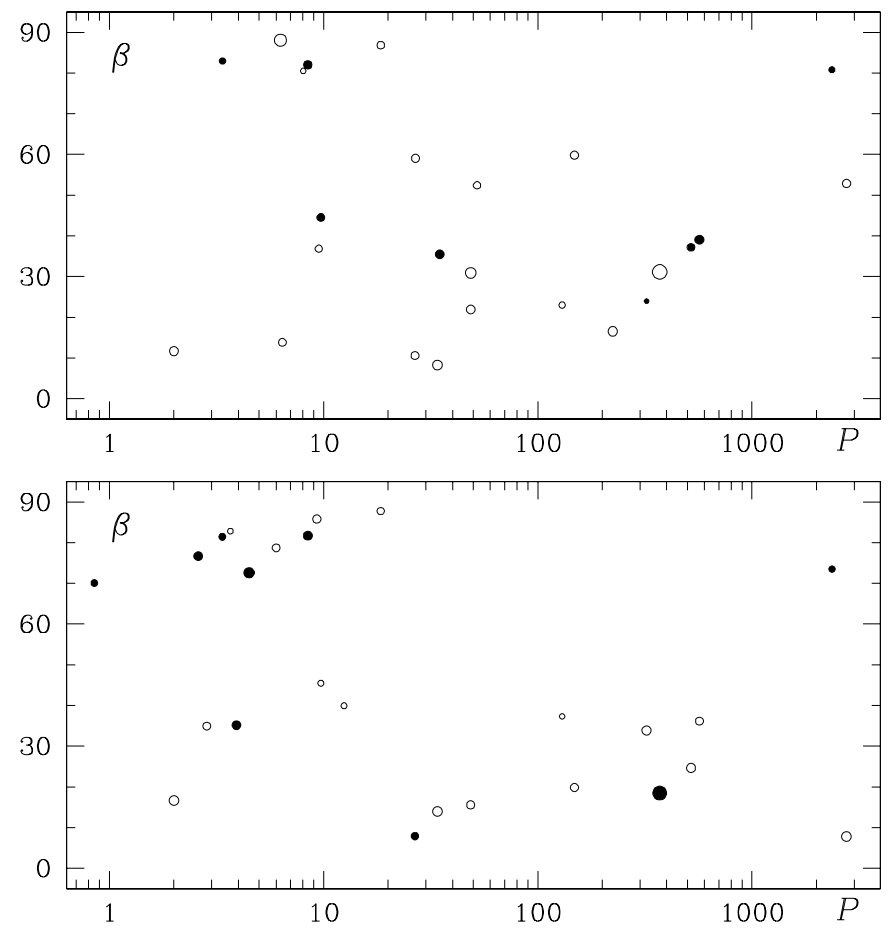

Fig. 24. Same as the lower panel of Fig. 16 for cases $p 1$ (upper panel) and $p 2$ (lower panel).

be of class II in the $p 1$-case and nearly of class II in the $p 2$-case, in accordance with the tentative "law" of Sect. 3.

On the whole, the existence of some correlation between $d$ and $P$ (small- $d$ models for short-period stars, large- $d$ models for long-period ones) seems somewhat related to the reliability of $\left\langle\mathcal{B}^{2}+\mathcal{B}_{z}^{2}\right\rangle^{1 / 2}$ observations, being more likely if they were not fully reliable, and vice versa. In any case, there is a considerable excess of models with $\beta_{\mathrm{Q}} \simeq 90^{\circ}$ and/or $\gamma_{\mathrm{Q}} \simeq 90^{\circ}$ : this comes out clearly from all three samples (see Figs. 5, 20, and 21).

As for the angles $\chi_{-}$and $\chi_{+}$, they seem to be uncorrelated - as in the $g$-case - with the rotational period. For the angle $\beta$, characterising the dipole orientation, we found the results presented in Fig. 24. They roughly confirm, though with a larger scattering, the behaviour deduced from the $g$-case (cf. Fig. 16): large $\beta$ values for short-period stars, small $\beta$ values for longperiod ones.

\section{Discussion and conclusions}

We have carried out a study of the magnetic morphologies of some thirty CP stars, by interpreting the observations of mean longitudinal field, crossover, mean quadratic field, and mean field modulus in terms of a magnetic dipole plus non-linear quadrupole model.

In most of the cases we could not obtain a unique model, even for those stars whose magnetic geometry seems strongly constrained by the observations. This is related to the relatively large observational errors, and possibly to the fact that the actual magnetic geometry of CP stars is too complex to be described in terms of a second-order multipolar expansion. We thus decided to follow an approach that gives a limited importance to the best-model of the individual stars, searching instead for characteristics that emerge from the whole set of models (corresponding to the absolute and relative minima of the $\chi^{2}$ hypersurface), and weighting the model parameters with the $\chi^{2}$ value.

Furthermore, we had to face the problem of the inconsistency of mean field modulus and mean quadratic field measurements: in fact, evidence exists that for many stars, either the former are systematically slightly overestimated, or the latter are systematically slightly underestimated. To deal with this problem, we repeated our analysis considering different observational datasets. We first performed a modelling considering all the kinds of measurement that were available for each individual star. Then we repeated the same analysis neglecting the observations of mean quadratic field; finally, we performed the analysis considering all available data but the observations of mean field modulus. Numerical simulations performed by Bagnulo, Mathys, \& Stift (in preparation) suggest that the technique used to determine the mean quadratic field tends to underestimate the measurement. In addition to these numerical simulations, another argument supports the view that measurements of the mean field modulus are likely more reliable than determinations of the mean quadratic field. Field modulus measurements rely directly on the basic physics of the Zeeman effect and, for simple lines such as the Fe II $\lambda 6149$ magnetic doublet, are for all practical purposes independent of radiative transfer effects (Mathys 1989, Sect. 4.2.1), in contrast to quadratic field determinations, which involve fairly gross approximations of the effects of the radiative transfer in the stellar atmosphere. Accordingly, one could be tempted to give more weight to the results obtained neglecting quadratic field measurements. We nevertheless decided to accept as reliable only those results that were recovered in all three kinds of analysis. These are as follows.

i) The dipolar strength is usually comprised in the interval $3-20 \mathrm{kG}$, with a peak at something less than $10 \mathrm{kG}$. The strength of the quadrupolar component is the same as, or larger than the dipole strength.

ii) The dipole and quadrupole amplitude, as well as the ratio of quadrupole to dipole amplitude, tends to decrease with increasing rotational period. Consistently, the phase-averaged mean field modulus, an indicator of the typical field strength, also tends to decrease with increasing $P$.

iii) The inclination of the dipole axis to the rotation axis is usually large for short-period stars, and small for long-period ones. This is in accordance with a recent result obtained by Landstreet \& Mathys (2000), who found a similar result for the magnetic axis of the axisymmetric model that they adopted in their best-fit technique.

iv) For several stars, the plane containing the two unit vectors that characterise the quadrupole is almost coincident with the plane containing the stellar rotation axis and the dipole axis. These are basically short-period stars (approximately, $P<10$ days). Long-period stars seem to be preferentially characterised by a quadrupole orientation such that the planes just mentioned are perpendicular. There is also some indication of a continuous transition between the two classes of stars with increasing rotational period, but the existence of such a "law" is 
partly related to the question of the reliability of mean quadratic field observations. This result is not recovered by the analysis performed neglecting the observations of mean field modulus (thus when quadratic field measurements have a larger weight).

It should be noted that the results of this analysis pertain to a sample of relatively slow rotating stars; many of them are in fact very long-period stars.

It is an unanswered question whether the magnetic field of $\mathrm{CP}$ stars is the relic of an interstellar field that became stronger and stronger as the primordial nebula collapsed into a star ("freezing" the field flux in a smaller and smaller plasma surface), or whether the field is at least in part dynamo generated. The results of our and previous studies show that there is some correlation between magnetic structure and rotational period, which encourages one to think in terms of the dynamo theory. In reality, it is not obvious to determine what is the consequence of what. For instance, Stȩpień \& Landstreet (2002) have argued that the interaction of the magnetic field and a circumstellar disk before the star reaches the Zero-Age-Main-Sequence tends to slow-down preferentially those stars that have magnetic field vectors tilted at a small angle with respect to the rotation axis (thus presumably with the field lines perpendicular to the disk). Therefore, the long rotational period would be a consequence of the magnetic structure, and the magnetic field itself could well be a fossil relic. On the other hand, finding within the framework of a fossil field an explanation for the statistical properties of the quadrupolar component that were found in this work may be less easy. In any case we still need firmer conclusions about the geometrical structures of magnetic $\mathrm{CP}$ stars, that could come for instance from extensive applications of Zeeman Doppler Imaging. At the same time, there is a strong need for a theory explaining the origin of the magnetic fields in A and B type stars capable of leading to predictions about the geometrical structures to be compared with the modelling results.

Acknowledgements. Many thanks to Pierre North for providing us with (unpublished) estimates of the radius for some of the stars of Table 1.

\section{References}

Bagnulo, S. 2001, in Magnetic fields across the Hertzsprung-Russel Diagram, ed. G. Mathys, S. K. Solanki, \& D. T. Wickramasinghe, ASP Conf. Ser., 248, 287

Bagnulo, S., \& Landolfi, M. 1999, A\&A, 346, 158

Bagnulo, S., Landi Degl'Innocenti, M., \& Landi Degl'Innocenti, E. 1996, A\&A, 308, 115
Bagnulo, S., Landolfi, M., \& Landi Degl'Innocenti, M. 1999, A\&A, 343, 865 (Paper II)

Bagnulo, S., Landolfi, M., Mathys, G., \& Landi Degl'Innocenti, M. 2000, A\&A, 358, 929 (Paper III)

Bagnulo, S., Wade, G. A., Donati, J. F., et al. 2001, A\&A, 369, 889

Borra, E. F., \& Landstreet, J. D. 1977, ApJ, 212, 141

Borra, E. F., \& Landstreet, J. D. 1978, ApJ, 222, 226

Hildebrandt, G., Scholz, G., \& Woche, M. 1997, Astron. Nachr., 318, 291

Hill, G. M., Bohlender, D. A., \& Landstreet, J. D., et al. 1998, MNRAS, 297, 236

Hubrig, S., North, P., \& Mathys, G. 2000, ApJ, 539, 352

Huchra, J. 1972, ApJ, 174, 435

Kochukhov, O., Piskunov, N., Ilyin, I., Ilyina, S., \& Tuominen, I. 2002, A\&A, 389, 420

Landolfi, M., Bagnulo, S., \& Landi Degl'Innocenti, M. 1998, A\&A, 338, 111 (Paper I)

Landstreet, J. D. 1992, A\&A Rev., 4, 35

Landstreet, J. D., \& Mathys, G. 2000, A\&A, 359, 213

Leone, F., Catanzaro, G., \& Catalano, S. 2000, A\&A, 355, 315

Leone, F., \& Catanzaro, G. 2001, A\&A, 365, 118

Mathys, G. 1989, Fundam. Cosmic Phys., 13, 143

Mathys, G. 1994, A\&AS, 108, 547

Mathys, G. 1995a, A\&A, 293, 733

Mathys, G. 1995b, A\&A, 293, 746

Mathys, G., \& Hubrig, S. 1997, A\&AS, 124, 475

Mathys, G., Hubrig, S., Landstreet, J. D., Lanz, T., \& Manfroid, J. 1997, A\&AS, 123, 353

Moss, D. 2001, in Magnetic fields across the Hertzsprung-Russel Diagram, ed. G. Mathys, S. K. Solanki, \& D. T. Wickramasinghe, ASP Conf. Ser., 248, 305

North, P. 1998, A\&A, 334, 181

Press, W. H., Teukolsky, S. A., Vetterling, W. T., \& Flannery, B. P. 1986, Numerical Recipes (Cambridge University Press, Cambridge)

Piskunov, N., \& Kochuckov, O. 2002, A\&A, 381, 736

Preston, G. W. 1969, ApJ, 157, 247

Preston, G. W., \& Stępień, K. 1968, ApJ, 151, 583

Stępień, K., \& Landstreet, J. D. 2002, A\&A, 384, 554

Strasser, S., Landstreet, J. D., \& Mathys, G. 2001, A\&A, 378, 153

Wade, G. A., Elkin, V. G., Landstreet, J. D., et al. 1996, A\&A, 313, 209

Wade, G. A., Elkin, V. G., Landstreet, J. D., \& Romanyuk, I. I. 1997, MNRAS, 292, 748

Wade, G., Donati, J.-F., Landstreet, J. D., \& Shorlin, S. L. S. 2000a, MNRAS, 313, 851

Wade, G. A., Kudryavtsev, D., Romanyuk, I. I., Landstreet, J. D., \& Mathys, G. 2000b, A\&A, 355, 1080

Wade, G. A., Debernardi, Y., Mathys, G., et al. 2000c, A\&A, 361, 991 